\title{
Spindle pole body-anchored Kar3 drives the nucleus along microtubules from another nucleus in preparation for nuclear fusion during yeast karyogamy
}

\author{
Romain Gibeaux, ${ }^{1,3}$ Antonio Z. Politi, ${ }^{1,3}$ François Nédélec, ${ }^{1,4}$ Claude Antony, ${ }^{1,4}$ and Michael Knop ${ }^{2,4}$ \\ ${ }^{1}$ European Molecular Biology Laboratory (EMBL), 69117 Heidelberg, Germany; ${ }^{2}$ Zentrum für Molekulare Biologie der \\ Universität Heidelberg (ZMBH), Deutsches Krebsforschungszentrum (DKFZ)-ZMBH-Allianz, 69120 Heidelberg, Germany
}

\begin{abstract}
Nuclear migration during yeast karyogamy, termed nuclear congression, is required to initiate nuclear fusion. Congression involves a specific regulation of the microtubule minus end-directed kinesin-14 motor Kar3 and a rearrangement of the cytoplasmic microtubule attachment sites at the spindle pole bodies (SPBs). However, how these elements interact to produce the forces necessary for nuclear migration is less clear. We used electron tomography, molecular genetics, quantitative imaging, and first principles modeling to investigate how cytoplasmic microtubules are organized during nuclear congression. We found that Kar3, with the help of its light chain, Cik1, is anchored during mating to the SPB component Spc72 that also serves as a nucleator and anchor for microtubules via their minus ends. Moreover, we show that no direct microtubule-microtubule interactions are required for nuclear migration. Instead, SPB-anchored Kar3 exerts the necessary pulling forces laterally on microtubules emanating from the SPB of the mating partner nucleus. Therefore, a twofold symmetrical application of the core principle that drives nuclear migration in higher cells is used in yeast to drive nuclei toward each other before nuclear fusion.
\end{abstract}

[Keywords: nuclear migration; microtubule; kinesin; karyogamy; spindle pole body; Kar3]

Supplemental material is available for this article.

Received September 19, 2012; revised version accepted December 27, 2012.

Microtubule (MT)-associated motor proteins play a major role in organizing MTs into dynamic structures that are essential for various segregation processes during cellular transitions or transport processes. Such molecular motors achieve their role by moving along MTs, by regulating the dynamical properties of MTs, and by interacting with other cellular structures (Mogilner and Craig 2010; Verhey et al. 2011). The MT minus end-directed motor Kar3 is a member of the nonprocessive kinesin-14 family and is required for different functions in mitosis, meiosis, and mating in yeast. In mitosis and, presumably, also prophase of meiosis I, Kar3 is required for a nonessential function in chromosome segregation by promoting normal kinetochore attachment of chromosomes to the spindle (Shanks et al. 2001, 2004; Jin et al. 2012) and for mediating anti-parallel interactions of interpolar nuclear MTs (Gardner et al. 2008). During mating, Kar3 plays an

\footnotetext{
${ }^{3}$ These authors contributed equally to this work.

${ }^{4}$ Corresponding authors

E-mail m.knop@zmbh.uni-heidelberg.de

E-mail antony@embl.de

E-mail nedelec@embl.de

Article is online at http://www.genesdev.org/cgi/doi/10.1101/gad.206318.112.
}

essential role in the formation of a diploid nucleus from the haploid nuclei of the mating partners.

The function of Kar3 is regulated by two different accessory proteins: Cik1 or Vik1 (Page and Snyder 1992; Page et al. 1994; Manning et al. 1999; Chu et al. 2005). Both Cik1 and Vik1 contain a central coiled-coil domain used for heterodimerization (Barrett et al. 2000). Cik1 is a nonmotor protein (Barrett et al. 2000), and Vik1 has a motor domain that lacks its ATP-binding site (Allingham et al. 2007). While Vik1 binds to Kar3 during vegetative growth and mitosis, it is not expressed during yeast mating (Manning et al. 1999). Cik1 is expressed during mitosis and mating, and the levels of both Kar3 and Cik1 are further increased upon exposure to pheromone (Meluh and Rose 1990; Page and Snyder 1992). Cik1 has a putative nuclear localization signal (NLS) at its $\mathrm{N}$ terminus that is important for the nuclear mitotic function of Kar3 in mitosis (Manning et al. 1999). During mating, a shorter cytoplasmic Cik1 protein lacking this NLS and an ubiquitylation site for the anaphase-promoting complex/cyclosome (APC/C) is generated by means of an alternative transcriptional start site (Benanti et al. 2009). This allows Cik1 to promote an essential cytoplasmic Kar3 function in mating 
by targeting it to cytoplasmic MTs and SPBs (Meluh and Rose 1990; Page et al. 1994).

After mating is initiated by cell-cell fusion, the haploid nuclei of the mating cells become transported toward each other in a process termed nuclear congression (Rose 1996). This involves specific regulation of MTs at the yeast centrosome, the spindle pole body ( $\mathrm{SPB})$. This matingspecific function of the SPB is initiated by mating signaling processes and leads to reorganization of the cytoplasmic MTs. Thereby, the $\gamma$-tubulin complex-binding protein Spc72, along with associated MTs, becomes released from its anchor protein, Nud1, at the outer plaque structure of the SPB and subsequently reappears at the half bridge appendage of the SPB. Here, it directly binds to the half bridge component Kar1, a tail-anchored membrane protein that is essential for mating and SPB duplication in vegetative cells (Rose and Fink 1987; Spang et al. 1995; Pereira et al. 1999). Upon establishment of cell-cell fusion at the site of cell polarity, the tip of the so-called shmoo, the half bridge-anchored MTs promote the migration of the two SPBs toward each other and thus of the entire nuclei. This process depends on the activity of the Kar3 motor protein.

To explain the mechanism of force generation for nuclear congression, two different models were proposed. Both models involve direct interactions between MTs from the opposite SPBs of the mating partners, however, with each having a different role for Kar3 (Molk and Bloom 2006). The "sliding cross-bridge" model (Rose 1996) proposed overlapping anti-parallel MTs that slide along each other and drive the nuclei toward each other via their SPB-anchored minus ends. In this model, Kar3 acts as cross-linker for anti-parallel MTs and provides by its minus end-directed motility the pulling forces to drag the two SPBs together (Polaina and Conde 1982; Meluh and Rose 1990; Endow et al. 1994). In addition, Kar3 was suggested to trigger shortening of opposing MTs at the SPBs (Rose 1996). An alternative model proposed direct interaction of MTs at their plus ends and their subsequent depolymerization. This is proposed to lead to a shortening of the MTs and, by this, drive nuclear congression. In this model, the Kar3/Cik1 heterodimer would function as a linker of the MT plus ends and induce depolymerization of the MTs from their plus ends, thus promoting the congression movements (Molk et al. 2006).

Upon completion of congression, nuclear fusion occurs in three steps in which each of the two bilayers of the nuclear membranes and, subsequently, also the SPBs fuse with each other (Melloy et al. 2007), giving rise to one nucleus containing a diploid genome and one so-called fusion SPB (Byers and Goetsch 1975). This process requires a set of additional Kar proteins for the membrane and SPB-related fusion processes (Melloy et al. 2009).

In this study, we used electron tomography, live-cell imaging, and simulation to quantitatively analyze the functional dynamics of MT interactions, MT anchor proteins, and the kinesin-14 Kar3 at high resolution. Our data suggest a new model for nuclear congression in which Kar3 is part of a module consisting of its light chain Cik1 and the $\gamma$-tubulin complex-binding protein Spc72, anchored to the nucleus via the half bridge component Karl of the yeast SPB. We show that this module underlies congression-specific MT organization. It anchors MT minus ends and, via Kar3 associated with the mating-specific Cik1 light chain, exerts the forces necessary for congression on MTs emanating from the SPB of the mating partner's nucleus. This permits the nuclei to move toward each other without the need of direct MT-MT interactions. Therefore, the Kar3-mediated migration of nuclei along MTs in yeast mating is analogous to nuclear migration processes in higher cells.

\section{Results}

\section{MT organization during nuclear congression}

Previous genetics and microscopic analyses led to the proposal of two models for how MTs could function to promote nuclear congression (Rose 1996; Molk et al. 2006). Hoping to distinguish between them, we imaged cells using electron tomography because its resolution in the range of 5-10 $\mathrm{nm}$ provides a precise snapshot of all MTs in the cell. We reconstructed wild-type cells that have undergone cell-cell fusion but in which the two nuclei were still separated, one on each side of the cell fusion pore $(n=$ 14 tomograms) (three zygotes shown in Fig. 1A; four other zygotes are shown in Supplemental Fig. S1A). In the segmented three-dimensional (3D) models, we observed distances between the two SPBs of the mating cells in the range of $0.16-2.57 \mu \mathrm{m}$. These values exhibited a good anticorrelation with the area of the openings between the cells (Supplemental Fig. S1B), indicating that the 3D models represent cells at different stages of congression, from early to late. Notably, inspection of the nuclear morphology identified 11 zygotes that exhibited protrusion-like nuclear deformations pointing toward the mating partner, each of which contained an SPB at the tip (Fig. 1A). We detected one to six MTs nucleated per SPB $3.12 \pm$ $1.24 \mathrm{SD}$ ). The origin of each MT was clearly indicated by the capped MT minus ends, which were found in close proximity (less than $\sim 15 \mathrm{~nm}$ ) to the half bridge of each SPB (Fig. 1B). This is consistent with the previous observation of MTs emanating from the half bridge upon pheromone stimulation in yeast (Byers and Goetsch 1975). The plus ends, characterized by their open morphology, were detected in the cytoplasmic space between and around the two nuclei.

The MT organization in the 14 zygotes, however, was not consistent with any of the published models because direct MT-MT interactions in the space between the two nuclei were dramatically underrepresented. Instead, MTs of variable length, with several of them much longer than the distance between the two SPBs, were observed. We thus scanned along MTs originating clearly from one SPB to identify whether they would come in close proximity to other subcellular structures, including opposite MTs.

In all 14 zygotes, we identified eight cases in which one MT plus end appeared close to the lattice of an opposite MT $(<100 \mathrm{~nm})$, but none of these MTs exhibited an extended anti-parallel alignment. In six out of these eight 
A
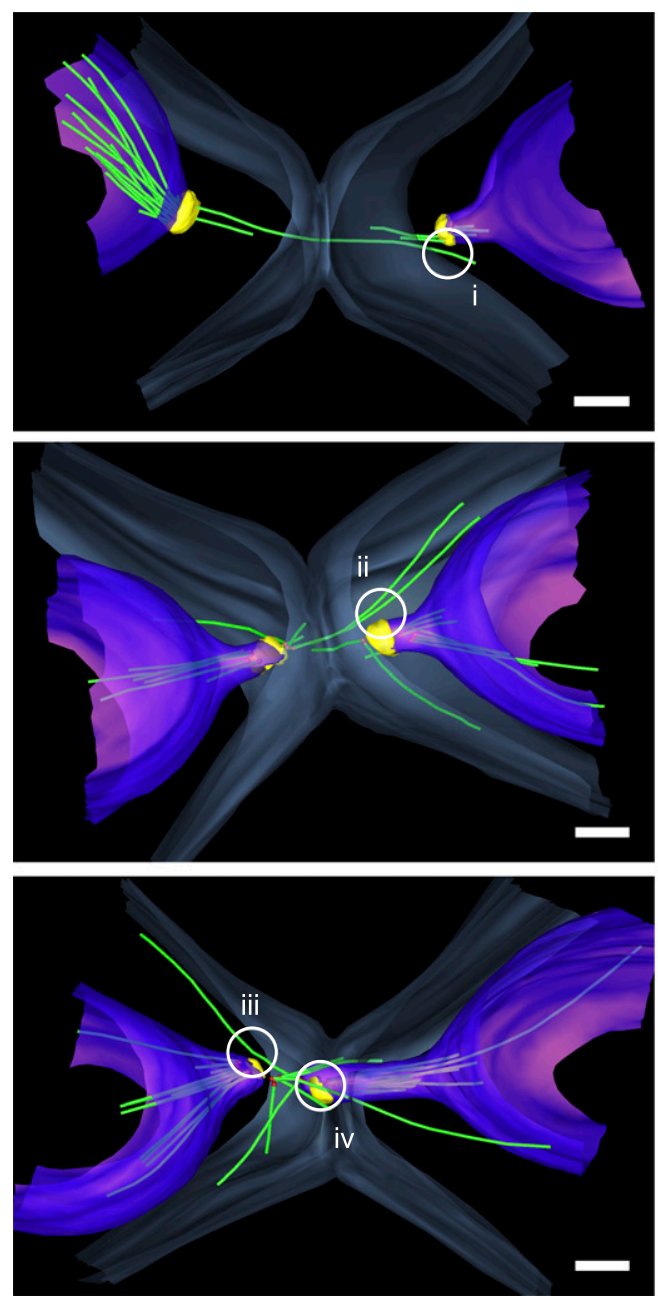

B

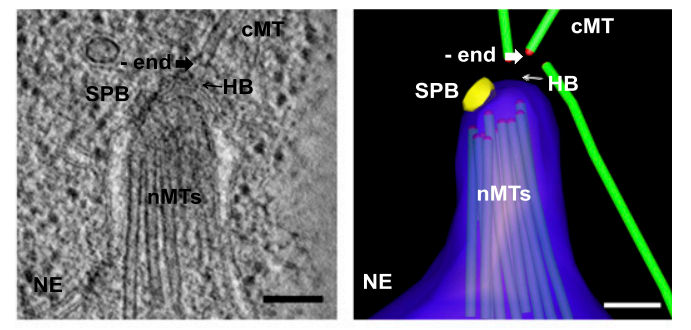

C
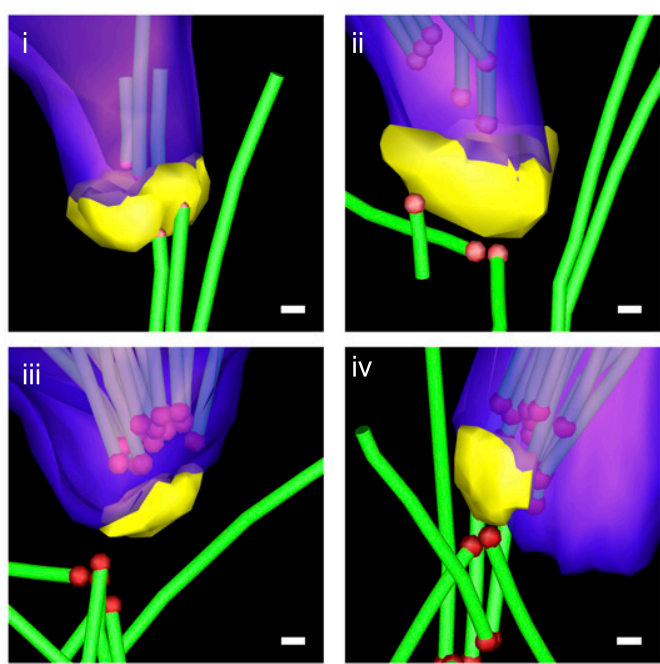

D

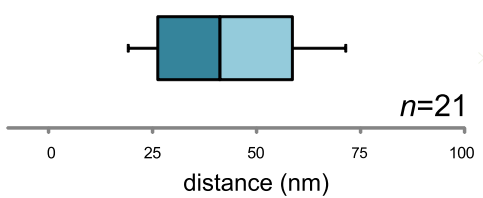

Figure 1. MT organization during nuclear congression reveals long MTs in the vicinity of opposite SPBs. $(A)$ Mating populations of wild-type (WT) yeast cells were cryofixed, freeze-substituted, and plastic-embedded. Electron tomograms of 300-nm sections were acquired from mating pairs. The panels show snapshots of the 3D-rendered situations of early, middle, or late stages (from top to bottom) of nuclear congression (Supplemental Movie S1), judged by the distances between opposite SPBs. (Light blue) Plasma membrane; (purple) nuclear envelope; (yellow) SPBs; (green) MTs. Bar, $300 \mathrm{~nm}$. (B) Electron tomographic slice and the corresponding area in the $3 \mathrm{D}$ model showing a SPB in a mating cell with half bridge-anchored MTs. Bar, $100 \mathrm{~nm}$. (C) Close-up view of four SPB models showing long MTs from the opposite SPB in their vicinity. Bar, $30 \mathrm{~nm}$. The distances were measured between these long MTs and opposite SPBs. (D) Distances between long MTs and SPBs. Median, upper, and lower quartiles (box) are shown.

zygotes with MT-MT adjacencies, the nucleus was strongly deformed, with the SPB located at the tip of a protrusion that extended toward the other nucleus. Furthermore, we observed 11 zygotes with one to four long MTs (two on average) that passed the opposite SPB in close proximity (Fig. 1C; Supplemental Fig. S1C; Supplemental Movie S1), with a median lateral MT-SPB distance of 58.8 $\mathrm{nm}(n=21)$ and a maximum distance of $106 \mathrm{~nm}$ (Fig. 1D). Interestingly, these 11 zygotes were also the ones harboring nuclear protrusions, whereas the three remaining zygotes, which showed no protrusions, also clearly did not show MTs in close proximity of opposing SPBs.

Assuming that the nuclear protrusions constitute a signature for pulling forces applied by a MT to an SPB, the co-occurrence of protrusions and MT-SPB adjacencies could point to the SPBs as the sites that connect to MTs from opposite SPBs. In contrast, only six out of the 11 zygotes with protrusions, but also two of the three nonprotruding zygotes, exhibited MT-MT adjacencies. Altogether, our observations suggest a new model for nuclear congression, in which no direct MT-MT interactions, but rather SPB-MT interactions, are necessary to generate forces during nuclear congression. In such a model, the forces pulling the nuclei together could be generated, for example, by a minus end-directed MT motor that is anchored on the outer surface of the SPB. The observed nuclear protrusions would also be a direct signature of the forces. 
Gibeaux et al.

\section{Long MTs from one SPB connect the opposite SPB during nuclear congression}

The observed median distance of $58.8 \mathrm{~nm}$ between a MT and its opposite SPB is comparable with the size of a kinesin motor protein (Howard 1996). The minus enddirected kinesin Kar3 is known to be essential for nuclear congression (Meluh and Rose 1990; Endow et al. 1994), and by using immunofluorescence microscopy in pheromone-stimulated cells, Kar3 was found to localize to MTs and their plus tips as well as to SPBs (Meluh and Rose 1990). To investigate the role of Kar3 during nuclear congression in living cells, we generated an endogenously expressed and fully functional $\mathrm{N}$-terminally tagged GFPKar3 fusion protein (Supplemental Fig. S2A). GFP-Kar3 localized to the SPBs labeled with Spc72-3mCherry throughout nuclear congression. In addition, GFP-Kar3 also localized to the lattice of MTs and at MT plus ends, in agreement with previous data from immunofluorescence microscopy (Fig. 2A; Supplemental Movie S2; Meluh and Rose 1990). Consistent with the observations made by electron tomography (Fig. 1), we also detected long MTs that appear to extend across the gap between the two nuclei beyond the opposing SPBs (Supplemental Fig. S2B). This shows that long MTs nucleated at one SPB pass in close proximity and beyond the opposite SPB. Therefore, Kar3, localized at the opposite SPB, could act laterally on these MTs to generate the pulling forces necessary for nuclear congression. Furthermore, we quantified the dynamics of nuclear congression using Spc42GFP-labeled SPBs. Cytoplasmic TagBFP expressed in one mating partner was used as a proxy for cell-cell fusion (Fig. 2B). The plots of the distance between the SPBs as a function of time after cell-cell fusion $(n=30)$ (Fig. 2C) revealed three distinct phases of nuclear congression. In phase $i$, the SPBs move little, while in phase ii, they approach each other with a speed of $1.27 \pm 0.35 \mu \mathrm{m} / \mathrm{min}$. In 11 out of 30 traces, no clear phase i could be observed, and the SPBs seemed to migrate almost immediately after cell-cell fusion. Finally, in phase iii, the SPBs remain close to each other at a distance of $<1 \mu \mathrm{m}$ for a variable period of time ( 22 out of 30 cells). Completion of nuclear congression, indicated by a merge of the two SPB signals, was detected in 24 out of the 30 cells investigated within the
A
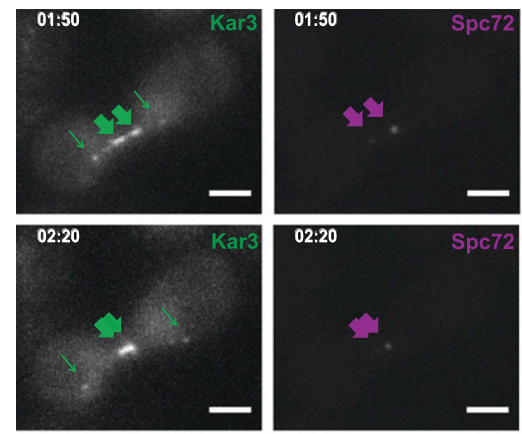

B
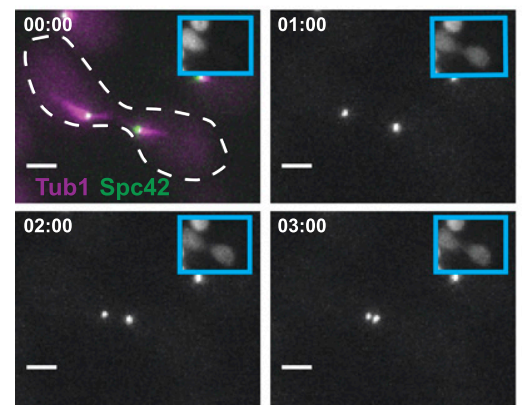

C

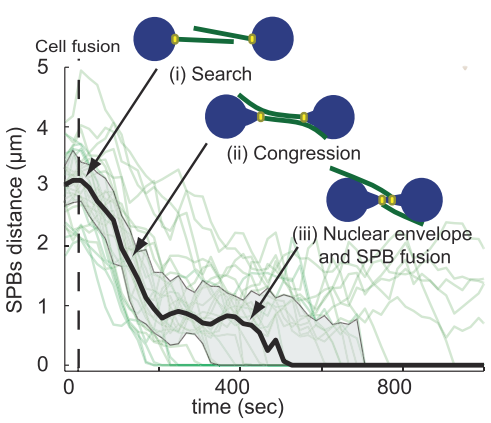

D
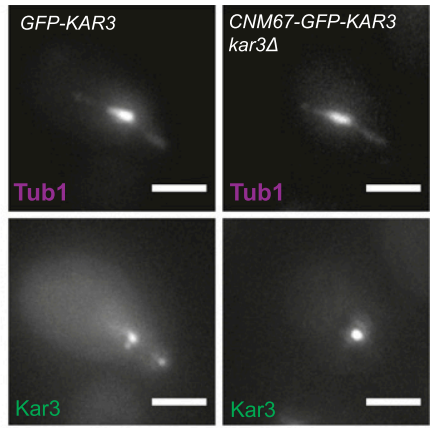

E
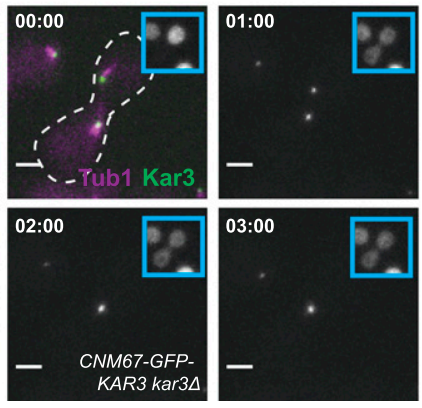

F

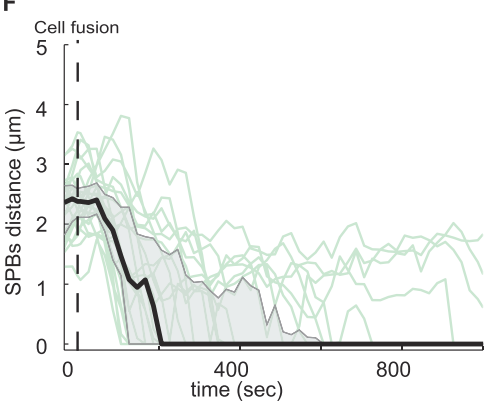

Figure 2. SPB-localized Kar3 promotes nuclear congression. (A) Localization of GFP-Kar3 to MTs and the SPB and of Spc72-3mCherry to SPBs observed by wide-field microscopy in mating cells (thick arrows point to the SPBs; thin arrows point to the plus end of the long MTs) (Supplemental Movie S2). Bar, $2 \mu \mathrm{m}$. (B) SPB congression during wild-type (WT) mating. The position of the SPBs was followed using Spc42-GFP (shown for all four time points), cytoplasmic TagBFP in one of the mating partners was used as a proxy for cell-cell fusion (inset), and mCherry-Tub1 was used to monitor MTs (shown only for the first time point). (C) Dynamics of nuclear congression $(n=30)$. We distinguish three phases (shown in the top schemes). The solid thick black line is the median, and in this and subsequent panels, the shaded area is the area between the upper and lower quartile. Green lines are the individual mating trajectories. (D) Comparison of Cnm67GFP-Kar3 with GFP-Kar3 localization on MTs (mCherry-Tub1) in mating pheromone-stimulated $(2 \mathrm{~h})$ cells. The Cnm67-GFP-Kar3 was expressed from the chromosomal CNM67 promoter in a strain deleted for the endogenous KAR3 gene. (E) Localization of GFP-Kar3 and MT in MATa and MAT $\alpha$ mating cells, both CNM67-GFP-KAR3 kar3A. Four time points during nuclear congression at intervals of 1 min are shown (MTs are only shown for the first time point) (Supplemental Movie S3). Bars, $2 \mu \mathrm{m}$. $(F)$ Dynamics of nuclear congression $(n=25)$ in CNM67-GFP-KAR3 kar3A cells. Cell-cell fusion was detected as in wild-type cells. 
$15 \mathrm{~min}$ of the image acquisition periods. This last phase may correspond to fusion of the nuclear membranes, followed by fusion of the nucleoplasms and finally of the SPBs (see also Fig. 5A in Melloy et al. 2007). Interestingly, the measured speed of congression matched the velocity of Kar3 (1-2 $\mu \mathrm{m} / \mathrm{min}$ ) measured in vitro (Endow et al. 1994; Chu et al. 2005).

Altogether, the results from electron tomography and live-cell imaging argue for a role of long MTs in promoting nuclear congression through interactions with the opposite SPB, possibly via Kar3.

\section{A cytoplasmic SPB-anchored pool of Kar3 promotes efficient nuclear congression}

The kinesin Kar3 is present at the SPB and along MTs. To distinguish the function of these two different pools of the motor protein during nuclear congression, we depleted Kar3 from the MT lattice only. To do so, we fused GFP-Kar3 directly to the $C$ terminus of the essential SPB outer plaque component Cnm67 (Muller et al. 2005). This alteration did not cause growth defects in cells still containing a wild-type copy of the KAR3 gene, indicating that Cnm67 remained functional and that the Cnm67-GFP-Kar3 fusion protein does not exhibit any dominant-negative effects in vegetative cells. Moreover, the Cnm67-GFP-Kar3 protein fusion did not rescue the slow-growth defect of a kar3s mutant, indicating that it does not complement the mitotic nuclear function of Kar3 (Supplemental Fig. S2C). We analyzed the intensity of the GFP-Kar3 signal on SPBs and along MTs using pheromone-treated CNM67-GFP-KAR3 kar3A cells. The Cnm67-GFP-Kar3 fusion protein was found to localize at the SPB, but in contrast to GFP-Kar3 cells, no signal was detected along MTs and in the cytoplasm (Fig. 2D; Supplemental Fig. S2D). Using DAPI staining to score nuclear fusion in mating cells, we found that the Cnm67GFP-Kar3 fusion was able to restore the mating defect of kar3s cells to $\sim 40 \%$ of the one observed for wild-type cells (Supplemental Fig. S2E).

We measured SPB congression dynamics in these cells (Fig. 2E; Supplemental Movie S3). In contrast to wild-type cells, we observed that all CNM67-GFP-KAR3 kar34 mating cells exhibited a clearly detectable phase $i$ of up to $200 \mathrm{sec}$ (Fig. 2F). Subsequent nuclear congression in phase ii, however, occurred with wild-type speeds $(1.45 \pm 0.42, n=25, P>0.05)$, but in the majority of the observed cases in the SPBs of the CNM67-GFP-KAR3 kar3 $\Delta$ cells, no clearly detectable phase iii was observed (16 out of 25) (Fig. 2F). Instead, the signals of the two SPBs directly merged with each other.

From these results, it appears that Kar3, when forced to localize to the outer plaque of the SPB, is able to exert motor protein activity sufficient to promote nuclear congression with wild-type speeds. However, initiation and termination of congression seem to occur in an altered manner. This may be caused by the absence of Kar3 at the MT lattice as well as the altered localization of Kar3 to the outer plaque instead of the half bridge of the SPB.
Cytoplasmic Kar3 localizes to the SPB through interaction with Spc72

Our results so far suggest that Kar3 is the likely candidate for the interaction of SPBs with cytoplasmic MTs from opposite SPBs. Interestingly, Kar3 localization to the SPB in pheromone-stimulated cells was shown to be dependent on Kar1 (Vallen et al. 1992), implying that Kar3 would localize at the cytoplasmic side of the SPB. However, in mitotic cells, Kar3 was found on the nuclear side of the SPB (Zeng et al. 1999). Therefore, to confirm that Kar3 indeed localizes at the cytoplasmic side of the SPB during wild-type mating, we used pheromone-stimulated cells and measured the position of the GFP-Kar3 signal relative to the SPB central plaque marker Spc42-mCherry using light microscopy. Gaussian fitting was used to detect the origin of the GFP-Kar3 fluorescence with subpixel accuracy. Moreover, Spc72-GFP and Spc110-GFP, which are known to localize at the cytoplasmic and nuclear sides of the SPB, respectively, were used to validate this approach (Supplemental Fig. S2F,G). Indeed, we found that Kar3 localizes at the cytoplasmic side of the SPB during mating (Fig. 3A).

We next used fluorescence recovery after photobleaching (FRAP) to investigate Kar3 dynamics at the SPB and obtained a mean residence time of GFP-Kar3 at the SPB of $15 \mathrm{sec}$ (Fig. 3B). Kinesin-14 minus end-directed and nonprocessive motor proteins, such as Kar3, bind transiently to MTs with a typical residence time $<200 \mathrm{msec}$ (Chen et al. 2011). The much longer residence time of Kar3 at the SPB thus likely reflects that the localization mechanism is independent of the binding activity of Kar3 to MTs. This was already suggested by the SPB localization of a truncated Kar3 fragment lacking the motor domain in pheromone-stimulated cells in the presence of nocodazole (Meluh and Rose 1990). To identify a possible molecular anchor of Kar3 at the cytoplasmic side of the SPB, we screened for two-hybrid interactions of Kar3 with known SPB components (Elliott et al. 1999). We detected an interaction between Kar3 and the C-terminal coiled-coil domain of Spc72 but not with the N-terminal domain (Fig. 3C) through which Spc72 binds to Spc97 and Spc98 of the $\gamma$-tubulin complex. This latter interaction of Spc72 underlies the nucleation and SPB anchorage of MT minus ends (Knop and Schiebel 1997). In vegetative cells, Spc72 binds to Nud1 at the SPB outer plaque, whereas it binds to Karl at the half bridge during mating (Pereira et al. 1999; Grueneberg et al. 2000). Also, Cik1, the Kar3 light chain involved in mating and mitosis (Page and Snyder 1992), was found to interact with Spc72 (Fig. 3C). Using kar3s, cik1s, and vik1s deletion mutants for two-hybrid testing, we observed a mutual dependency of Cik1 and Kar3 for their interaction with Spc72, whereas Vik1 was not required (Fig. 3C). This is fully consistent with the observation that Cik1 localization to SPBs in pheromone-treated cells depends on Kar3 (Benanti et al. 2009), and the localization of Kar3 to the cytoplasmic side of the SPB depends on Cik1 (Fig. 3D), as also previously suggested by immunofluorescence analysis (Page et al. 1994). 

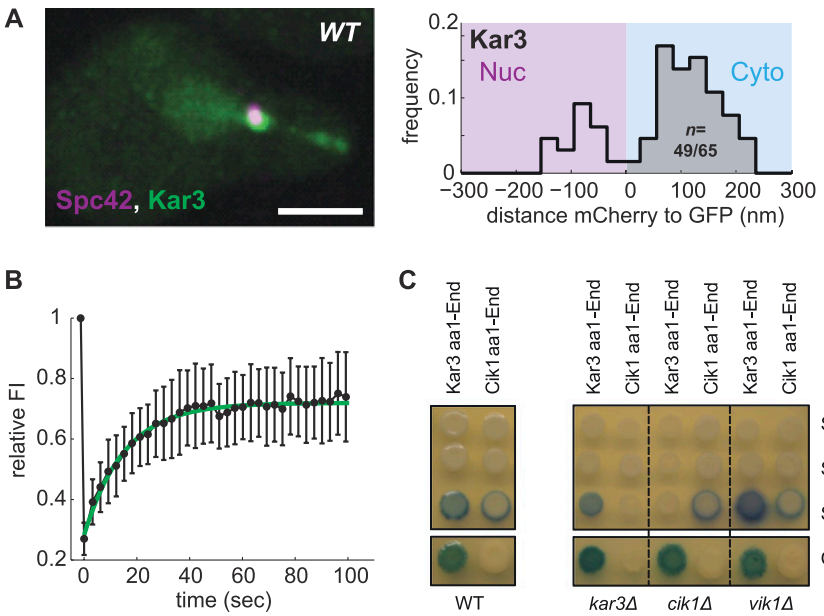

C
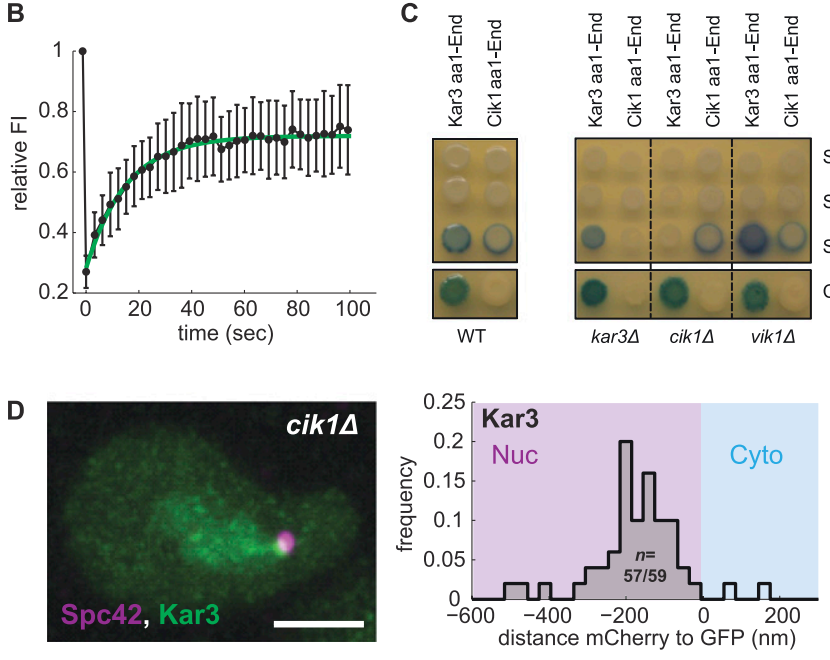

These results indicate a dual role of Spc72 at the half bridge of the SPB for the interaction with MTs during nuclear congression. Spc72 binds MT minus ends via the $\gamma$-tubulin complex (Pereira et al. 1999) and simultaneously also anchors the Kar3/Cik1 complex.

\section{Kar1-anchored Spc72/Kar3 machinery is sufficient to promote MT minus end movements}

The SPB half bridge component Karl is anchored with its C-terminal membrane domain in the nuclear envelope (Spang et al. 1995), whereas a region in the N-terminal half (amino acids 116-236) interacts directly with Spc72 (Pereira et al. 1999). kar1 mutations in the Spc72 in-

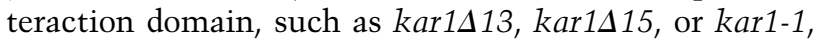
are the only ones known that lead to a unilateral mating defect (Conde and Fink 1976; Rose and Fink 1987; Vallen et al. 1992; Pereira et al. 1999) in which it is sufficient to have the mutation in only one of the two mating partners to abrogate mating completely (to the level observed when both partners contain the mutation). Our finding that Spc72 binds Kar3/Cik1 in addition to MT minus end-binding proteins is consistent with this. Presumably, such kar1 mutants do lose simultaneously both types of SPB-MT interactions. Thereby, MTs nucleated and anchored at the wild-type $K A R 1$ SPB fail to interact with the kar1 SPB, which itself is also unable to anchor and nucleate MTs.

To test this model further, we used live-cell microscopy. Imaging GFP-Kar3 in kar1 15 cells, we indeed observed the loss of cytoplasmic, but not nuclear, GFP-Kar3 signal from the SPB, along with the detachment of Spc72 and the anchored MTs, upon stimulation with pheromone (Fig. 4A-C; Supplemental Movie S4). This is consistent with
Figure 3. Interaction of Kar3/Cik1 with Spc72. (A) Localization of Kar3 to the cytoplasmic side of the SPB. Fluorescence images of pheromone-stimulated cells (left) expressing GFP-Kar3 and Spc42-mCherry were analyzed, and the distances between the center of the GFP-Kar3 and Spc42-mCherry signals were measured and plotted in a histogram relative to the Spc42-mCherry signal $(n=$ 65 cells) (right). Forty-nine cells exhibited the signal on the cytoplasmic side of the SPB (labeled Cyto). Considering that the SPB is subjected to dynamic rapid movements due to MT-exerted forces, we account this as confirmation of the presence of cytoplasmic pools of Kar3 in pheromone-stimulated cells. The image has been filtered with a Gaussian filter of radius 1. (B) Dynamic binding of GFP-Kar3 to the SPB in pheromone-stimulated cells. FRAP estimated a recovery rate of $0.0663 \pm$ $0.0375 / \mathrm{sec}$. (Black line) Mean and standard deviation of data $(n=30)$; (green line) exponential fit to the mean. (C) Two-hybrid interactions of Kar3 and Cik1 with fragments of Spc72 and the N-terminal half of Cik1 in wild-type (WT) and kar3s, cik1s, and vik1s mutant strains. $\beta$-Galactosidase activity was determined using a plate assay. Blue colony color indicates interaction. (D) Accumulation of GFP-Kar3 at the nuclear side of the SPB (labeled Nuc) in cik1s cells. Experimental conditions are as in $A$. GFP-Kar3 was detected at the nuclear side of the SPB in 57 cells $(n=59)$. Bar, $2 \mu \mathrm{m}$. previous observations showing that a motor-less domain of Kar3-lacZ fusion protein does not bind to kar1413 SPBs in pheromone-stimulated cells (Vallen et al. 1992).

To investigate the nuclear morphology and the MT organization as a function of Spc72 binding to the half bridge, we generated tomograms from zygotes of kar1 15 mated to wild-type cells $(n=8)$ (Supplemental Movie S5). This always revealed one SPB completely free of MTs minus ends-likely the kar1 15 -containing SPBs (Fig. 4D). These SPBs were never observed in close proximity to MTs nucleated from the opposite-and presumably wild-type-SPB. Furthermore, nuclear protrusions were absent in these zygotes, suggesting that their formation depends on Kar1-anchored Spc72 and functioning nuclear congression (Fig. 4D). In addition, we observed free MTs in the cytoplasm ( $n=7$ in a total of 8 tomograms), as reported for pheromone-stimulated cells (Rose and Fink 1987). These MTs each exhibited a capped end, most likely the minus ends that detached from the kar1415 SPBs (Fig. 4D). Strikingly, most of the capped minus ends were detected in close proximity $(<100 \mathrm{~nm})$ to the lattice of a MT from the opposite SPB $(n=6$; median distance of $30.5 \mathrm{~nm})$ (Fig. 4E,F).

This result could suggest active capturing of these minus ends by other MTs. To investigate this possibility, we followed the fate of detached MTs in kar1415 strains using live-cell imaging and GFP-tubulin and Spc72-3mCherry as labels. In several cases, we observed capturing of a free end of a released MT by a MT of the opposite SPB $(n=20$ out of 22) (Fig. 4G,H). In all recorded events, the captured end was labeled with Spc72, and in the majority of the cases $(n=17$ out of 22), subsequent movement toward the minus end of the capturing MT (as indicated by the position of the opposite SPB) (Supplemental Movie S6) was observed. In some cases, we could follow their movement until they 
A

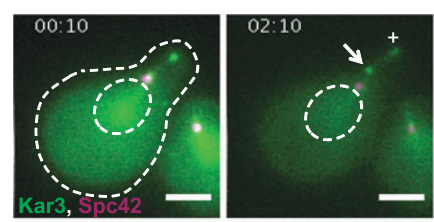

B

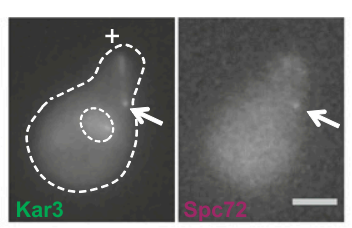

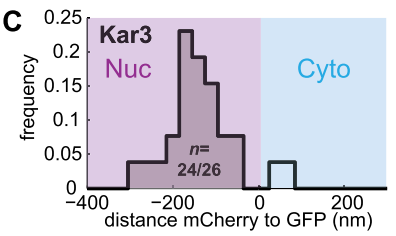

D

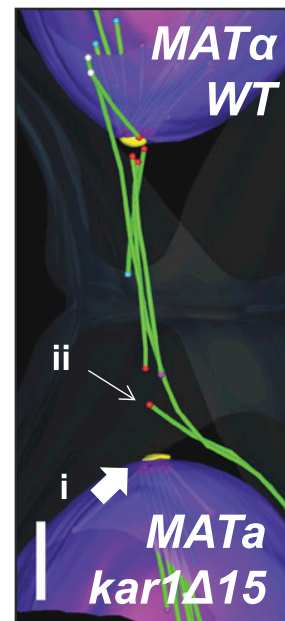

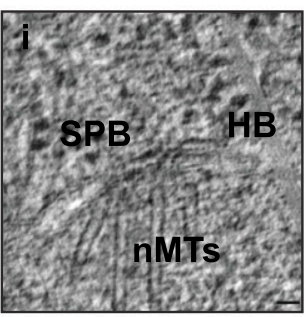

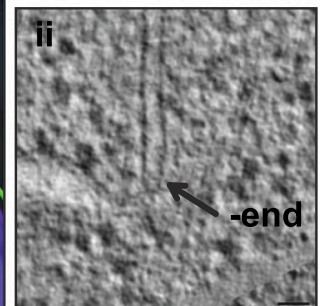

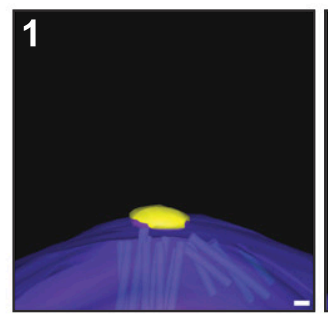

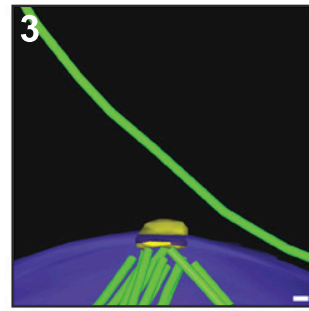

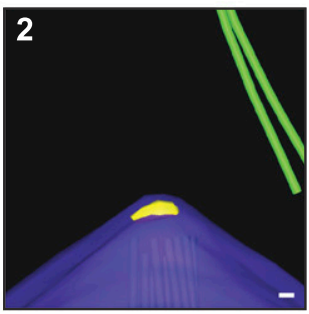

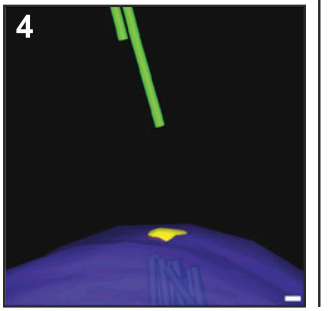

E
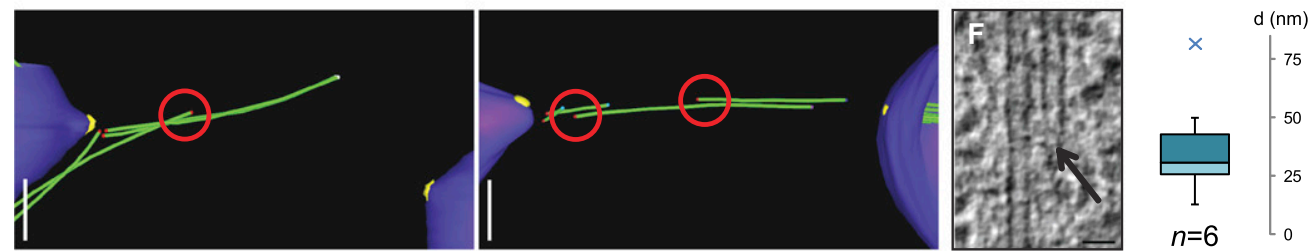

G
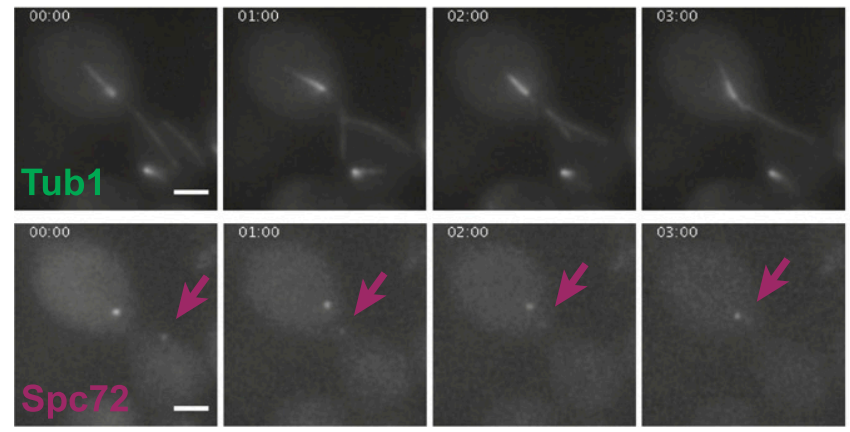

H
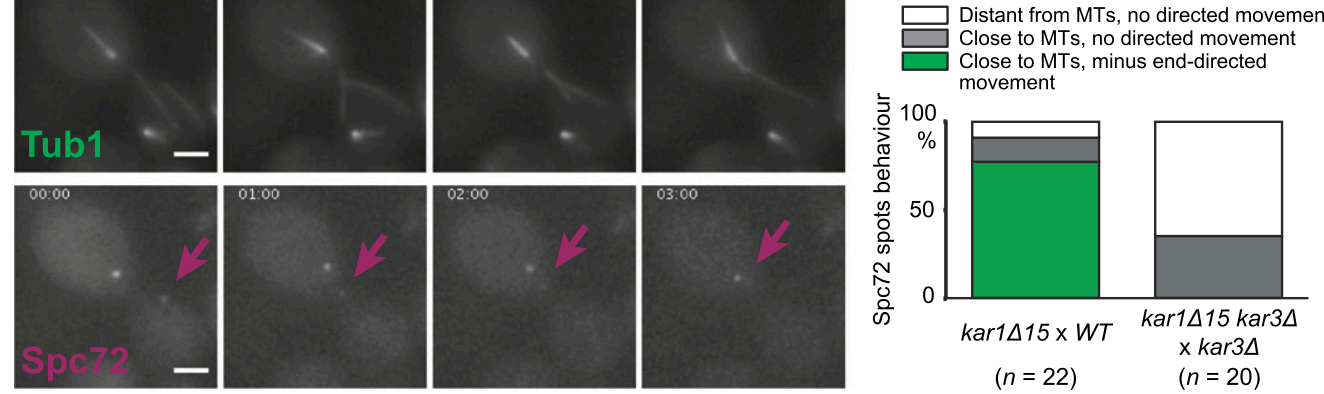

Figure 4. Karl-anchored Spc72/Kar3 provides MT minus end-directed mobility. $(A)$ Release of cytoplasmic MT together with a minus end-attached GFP-Kar3 pool from the SPB (followed with mCherry-labeled Spc42) in a pheromone-stimulated kar1415 cell (Supplemental Movie S4). The nucleus is indicated with the dashed line that limits the GFP-Kar3 nuclear signal, the arrow points to the detached MT minus end, and the plus sign $(+)$ identifies the plus end at the shmoo tip. Bar, $2 \mu \mathrm{m}$. (B) Released cytoplasmic MT harboring a minus end-attached GFP-Kar3 (left) and Spc72-3mCherry (right) pools detached together from the SPB in a pheromonestimulated kar1 15 cell. The nucleus is indicated with the dashed line that outlines the GFP-Kar3 nuclear signal, the arrow points to the detached MT minus end, and the plus sign $(+)$ identifies the plus end at the shmoo tip. Bar, $2 \mu$ m. $(C)$ SPB-retained GFP-Kar3 in pheromone-stimulated kar1 15 cells with detached cytoplasmic MTs localizes to the nuclear side of the SPB $(24$ cells, $n=26$, labeled Nuc). Experimental settings are as in Figure 3A. (D) 3D models of mating between MATa kar1 15 and MAT $\alpha$ wild-type (WT) partners. Typically, one SPB was found to be free of cytoplasmic MTs (kar1 $15 \mathrm{SPB})$, and released MTs with free capped minus ends are visible in the cytoplasm (Supplemental Movie S5). (Light blue) Plasma membrane; (purple) nuclear envelope; (yellow) SPBs; (green) MTs. Bar, $300 \mathrm{~nm}$. (Panels i,ii) Electron tomographic slices show the region around the indicated areas in the model to the left. Bar, $30 \mathrm{~nm}$. (Panels 1-4) 3D models show close-up views of different kar1 15 SPBs. Bar, $30 \mathrm{~nm}$. (E) 3D models of mating events between kar1415 and wildtype cells. (Red circles) The minus ends of detached MTs in the vicinity of another MT; (purple) nuclear envelope; (yellow) SPBs; (green) MTs; (red dots) MT minus ends. Bar, $300 \mathrm{~nm}$. $(F, l e f t)$ Electron tomographic slice showing a capped minus end of a detached MT in the vicinity of another MT. Bar, $30 \mathrm{~nm}$. (Right) Distances between detached MT minus ends and the lattice of the closest MT. Median, upper, and lower quartiles (box) are shown. Individual outliers (cross) are shown if above/below quartile $+1.5 \times$ (interquartile range) (whiskers). (G) Frames from a movie showing the capture and alignment of a detached MT in a kar1s15 to a MT from the opposite wildtype SPB. GFP-Tub1 (left) and Spc72-3mCherry (right) were detected (Supplemental Movie S6). (H) Behavior of Spc72 dots during mating in wild-type and mutant cells. Spc72-3mCherry- and GFP-tubulin-labeled cells of both mating types (with mating type-specific genotypes, as indicated) were mixed to allow mating to occur followed by movie recording in cells undergoing mating. The behavior of Spc72-3mCherry was quantified with respect to detachment from one of the two SPBs (presumably the kar1 15 SPB) and its position and movement were quantified with respect to cytoplasmic MTs that emanate from the SPB with attached Spc72. 
reached a parallel alignment with the capturing MT. The observed minus end-directed movement suggests the presence of active Kar3 motor protein, together with Spc72, at the minus ends of the released MTs. Indeed, in a kar34 background, none of the Spc72-containing MTs showed minus end-directed movements along other MTs (Fig. 4H). These results indicate that the detached Kar3/Spc72 module at MT minus ends in kar1 15 cells is fully functional, thereby implying that Kar3 bound to Spc72 at the half bridge in wild-type cells is active and able to promote minus end-directed movements.

\section{An asymmetric MT organization is sufficient for nuclear congression}

It was previously reported that the overexpression of Spc72 in kar1-1 mutant cells partially rescued mating with wild-type cells (Fig. 5A; Pereira et al. 1999). In vegetative cells, overexpression of Spc72 led to the formation of a large aggregate of the protein at the SPB outer plaque (Souès and Adams 1998) and an increased number of cytoplasmic MTs (Knop and Schiebel 1998). Whereas Spc72 detaches from the SPB in kar1d15 mutants stimulated with pheromone (Pereira et al. 1999), we observed persistent binding in the case in which Spc72 was overexpressed, along with attached MTs (Supplemental Fig. S3A). This could explain how Spc72 overexpression in kar1415 cells can rescue the mating defect. Interestingly, the mating defect was even more efficiently rescued when Spc72 was overexpressed in the wild-type instead of the kar1415 mating partner (Fig. 5A; Supplemental Fig. S3B-D). We used electron tomography to investigate this further. We observed a significant increase of the MT number at the wild-type SPB ( $n=3$ ) (Fig. 5B), all of which appeared to be nucleated from aggregates of Spc72 at the SPB outer plaque (Fig. 5C). In contrast, we did not observe MTs nucleated from the kar1 15 SPB (Fig. 5D). Nonetheless, we noticed in one tomogram a cell in an advanced state of nuclear congression, as indicated by the close proximity of the two SPBs from facing nuclei, in spite of the absence of MTs at one SPB (Fig. 5D, panel ii). In this tomogram, the nucleus containing the anchored MTs exhibited a characteristic nuclear protrusion otherwise found only in wildtype tomograms but never in kar1s15 zygotes outside the overexpression context. This seemingly contradicted the idea of Spc72 being the sole anchor at the SPB for the machinery that mediates lateral MT interactions.

To further investigate how Spc72 overexpression in the wild-type partner of a wild type $\times$ kar $1 \Delta 15$ mating could rescue mating with an asymmetric $\mathrm{MT}$ organization, we analyzed MT dynamics in mating cells. To distinguish the genotypes of the mating partners, we labeled Spc72 with $3 \mathrm{mCherry}$. Before cell-cell fusion, the overexpressing cell showed an increased cytoplasmic fluorescence. As suggested by electron tomography, this revealed nuclear congression that appeared to progress with MTs nucleated only at the SPB of the Spc72-overexpressing cell (Fig. 6A; Supplemental Movie S7). In addition, however, we observed that small but detectable amounts of Spc72 localized at the SPB of the kar1415 cell after cellcell fusion, while no MTs were found to emanate from this SPB (Fig. 6B). It is therefore likely that Spc72 at the
A

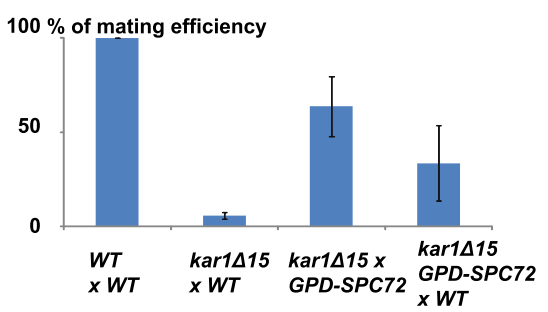

B

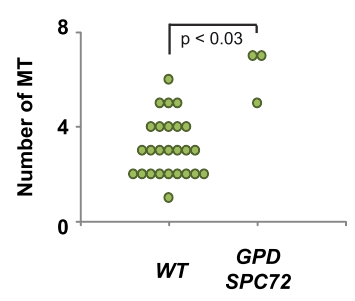

C
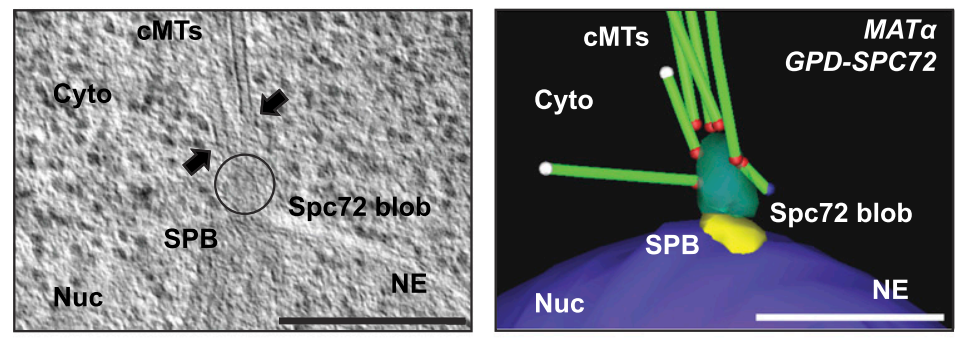

D
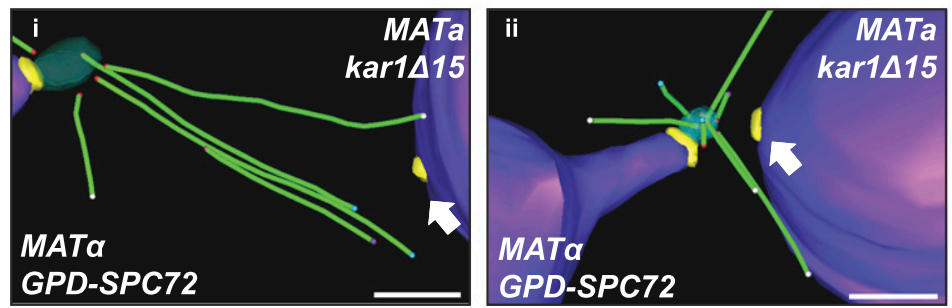

Figure 5. Overexpression of Spc72 in wild-type

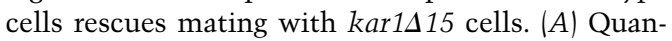
tification of the mating efficiency of kar1s15 cells mated to wild-type (WT) cells as a function of overexpressed Spc72, relative to the wild-type mating efficiency (mean $\pm \mathrm{SD}$ ). Genotype of mating partners as indicated in the legend. $(B)$ Quantification of cytoplasmic MTs nucleated from wild-type and SPC72-overexpressing SPBs from electron tomograms $(n=26$ and $n=3)$. The $P$-value was determined using a two-tailed $t$-test. $(C)$ Slice from electron tomogram (left) and the corresponding model (right) of a SPB in the Spc72-overexpressing

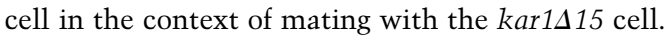
Bars, $300 \mathrm{~nm}$. (D) Electron tomography 3D models of early (left) and late (right) stages of nuclear congression of nuclei with the indicated genotypes. (Purple) Nuclear envelope; (yellow) SPBs; (green) MTs. Bars, $300 \mathrm{~nm}$. 
A

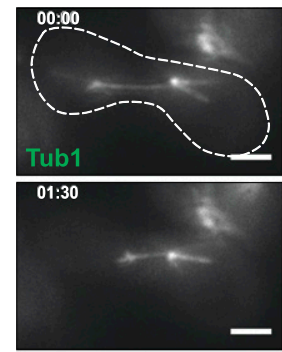

B

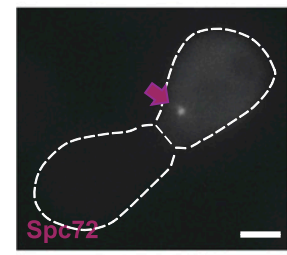

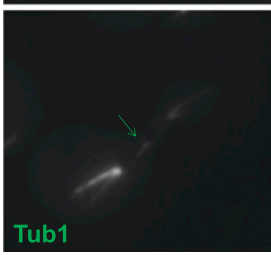

Before fusion
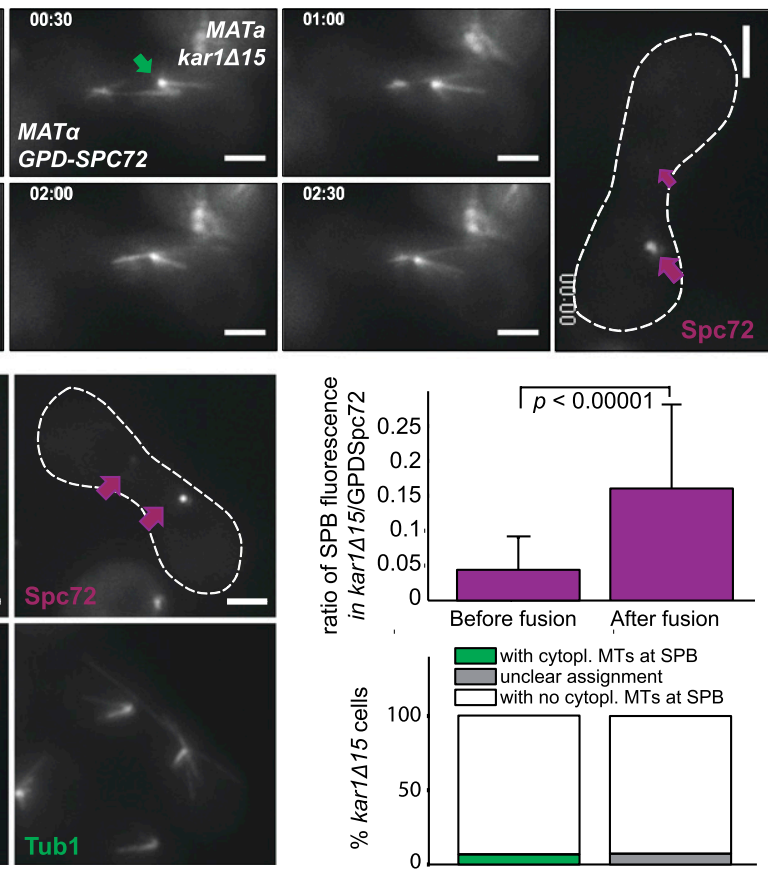

After fusion

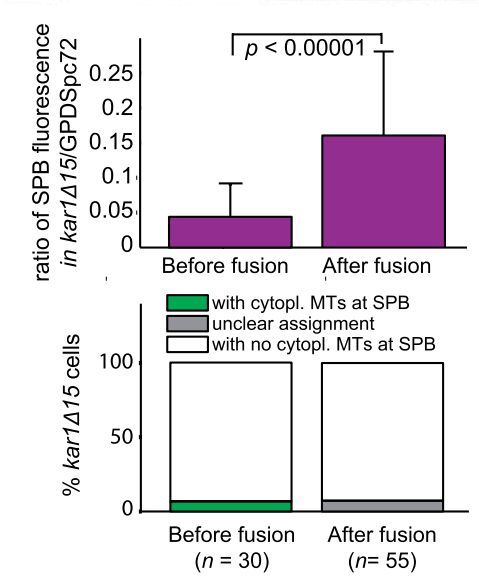

Figure 6. Asymmetric MT organization and movement of one SPB toward the other SPB. (A) MT dynamics during nuclear congression in a MATa kar1 15 cell mated to a MAT $\alpha$ GPD-SPC72 cell. Images at intervals of $30 \mathrm{sec}$ are shown (Supplemental Movie S7). MTs were detected using GFP-Tub1 (left), and the SPBs were detected using Spc72-3mCherry (right). Note the absence of cytoplasmic MTs at the kar1 15 SPB (thick arrow). Bar, $2 \mu \mathrm{m}$. (B) Spc72-3mCherry signal before and after cell fusion during mating between kar1s 15 cells and wild-type (WT) cells with overexpressed SPC72. Spc72-3mCherry is expressed from its endogenous promoter in the kar $1 \Delta 15$ cell and overexpressed from the GDP promoter in the wild-type mating partner. The cytoplasmic mCherry signal is significantly higher in the cell with the overexpression construct. Cells were imaged as a single snapshot. Equilibration or nonequilibration between the two mating cells of the cytoplasmic Spc723 mCherry signal was used as a proxy to classify the cell snapshots into unfused/fused categories for quantification. Representative cells before and after cell fusion are depicted. Spc72-3mCherry and MTs are imaged as indicated. Magenta arrows indicate Spc72-

$3 \mathrm{mCherry}$ at the SPBs. Bar, $2 \mu \mathrm{m}$. Quantification of Spc72-3mCherry signal (top) and the detection of cytoplasmic MTs (bottom) at the kar1 15 SPB in cells imaged before or after cells fusion (mean \pm SD; the $P$-value indicates significance by a two-tailed $t$-test).

kar1 15 SPB would allow this SPB to interact with MTs of the opposite wild-type SPB through the recruitment of Kar3. Indeed, GFP-Kar3 could be found at the SPB cytoplasmic side in pheromone-treated kar1 15 cells overexpressing Spc72, despite the lack of attached cytoplasmic MTs (Supplemental Fig. S3E). Altogether, this suggests that the overexpression of Spc72 in wild-type cells is able to promote nuclear congression and mating by generation of an asymmetric MT organization with sufficient amounts of Kar3/Spc72 at the kard15 SPB. These motors are sufficient to promote migration of the kar $15 \mathrm{SPB}$ along MTs that are anchored at the opposite wild-type SPB. To further test this, we introduced the constitutively SPB-anchored Kar3 fusion protein Cnm67-GFP-Kar3 into a kar1 15 strain. This strain does not contain minus end-anchored MTs during mating with a wild-type partner. Nevertheless, we observed nuclear fusion with an efficiency similar to the one observed upon SPC72 over-

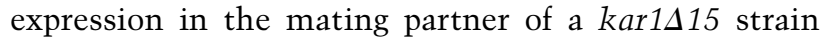
(Supplemental Fig. S3D). This again emphasizes that an asymmetric MT organization is sufficient for nuclear congression during mating. As long as one SPB provides MT anchorage and the other one provides the SPB-anchored Kar3 motor, nuclear congression is able to proceed. This also indicates that no direct MT-MT interactions are required for nuclear congression.

\section{In silico simulation of nuclear congression}

Altogether, our data suggest a model in which the minus end-directed motor Kar3 anchored at the cytoplasmic side of the SPB promotes nuclear congression by pulling the SPBs along the MTs of the mating partner. To test whether this is physically plausible, we simulated the process within accurate $3 \mathrm{D}$ geometry using the Cytosim platform (http:// www.cytosim.org) (Nedelec and Foethke 2007). The simulation is based on molecular descriptions of MT dynamics, the interactions of the Kar3/Cik1 dimer with MTs via its motor and nonmotor domain, and the SPB via its nonmotor domain (Fig. 7A,B). To complete the list of parameters required for the simulation (see Supplemental Table S1), we measured the number of Kar3 motors at the SPB in pheromonestimulated cells. The GFP-Kar3 fluorescence at the SPB in mating wild-type cells was quantified and calibrated using the fluorescence of signal of the kinetochore component Nuf2-GFP as a reference (Joglekar et al. 2006; Lawrimore et al. 2011). This estimated an average of $143 \pm 69$ GFPKar3 at the SPB (mean \pm SD, $n=34$ ) (data not shown).

We simulated the system for $20 \mathrm{~min}$ after cell-cell fusion (Supplemental Movie S8) and extracted the trajectories of the SPBs as a function of time.

Three models were simulated: Model 1 contained Kar3 only at the SPBs, model 2 contained Kar3 at the SPBs and the plus ends of MTs, and model 3 contained only MT plus end-localized Kar3 (Fig. 7A). We observed robust nuclear congression for simulations of models 1 and 2 (Fig. 7C). For model 3, the simulation did not lead to nuclear congression. A comparison of the trajectories from the simulations with wild-type live-cell imaging data (Fig. 2B) identified the best similarity of these data with the results obtained from model 2, where Kar3 localizes to SPBs, and a moderate number (three to five) 
of freely diffusible Kar3/Cik1 is found at the plus ends of MTs (Fig. 7C; Supplemental Movie S9). Model 1 yielded, with the same set of dynamic parameters, trajectories in agreement with the live-cell imaging data obtained from CNM67-GFP-KAR3 kar3A cells that do not contain MTlocalized Kar3, including a prolonged phase i (Figs. 2F, 7D). Therefore, the results from models 1 and 2 could point to a function of MT-localized Kar3 that promotes a shortening of phase i, possibly by orienting MTs toward the other SPB. In this model, we also tested the effect of two different amounts of Kar3 at the SPB and found that this only marginally influences the length of phase $i$ and had no impact on the speed of congression (Fig. 7D).

We next analyzed the organization of MTs in the different models with respect to the localization of the plus ends of

A

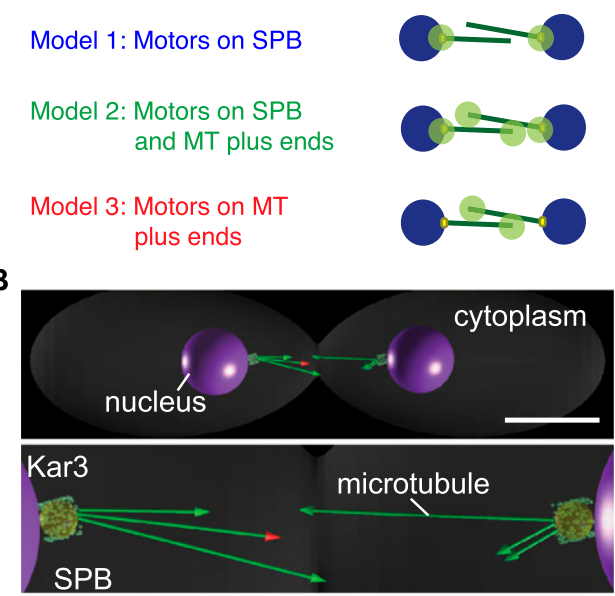

C

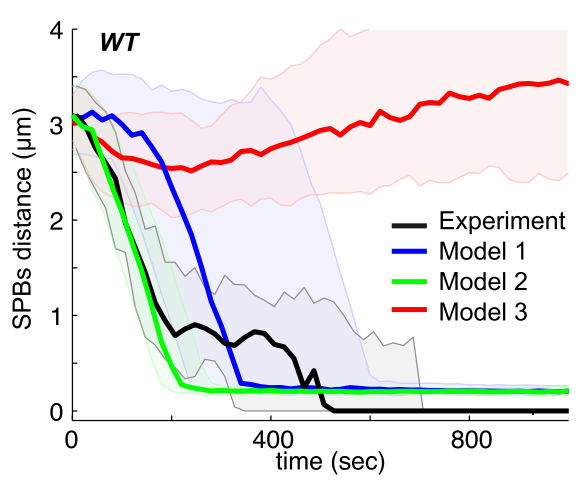

D

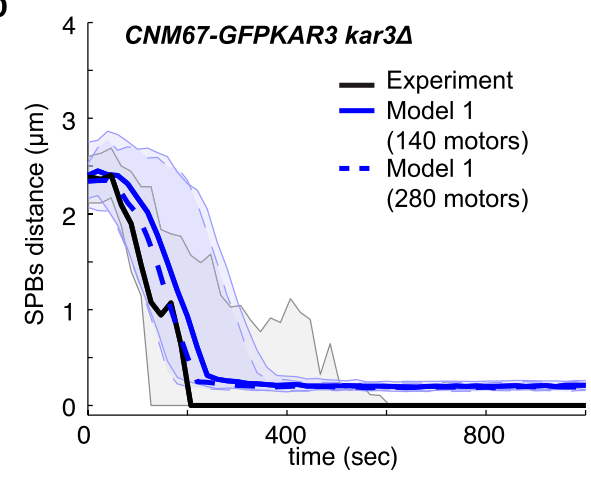

the MTs. In the absence of Kar3 at the MTs (model 1), we detected, on average, $30 \%$ of the cells at any given time point where a MT plus end was close to a MT of the opposite SPB. In model 2, we found that this value exhibited a peak with $64.4 \%$ of such cells $30-50$ sec after cell-cell fusion. Later on, this percentage returned back to a value of $30 \%$ as observed in model 1. This indicates that the observed shortening of phase $i$ in model 2 when compared with model 1 may be due to MT plus end-localized Kar3 motors interacting with the lattice of MTs originating from the opposite SPB. The frequency of such events in the simulation is furthermore consistent with the observation of such events in eight of the 14 tomograms (Fig. 1; Supplemental Fig. S1).

Phase iii, corresponding to a pausing phase prior to final SPB and nuclear fusion, was not covered by any of the simulations. This is due to our simplified description of MT anchorage at the SPB and the missing information about SPB substructures and membrane-related events that occur in the last steps of nuclear congression and fusion.

We convolved the simulation data to simulate live-cell imaging of cytoplasmic MTs and make the model directly comparable with experimental data (Supplemental Fig. S4). This led to images highly reminiscent of the ones observed previously (Molk et al. 2006), in which MT-MT overlap zones between the SPBs are absent. Thus, our model is also consistent with these data, although it differs from the model published together with them.

In conclusion, the stochastic simulations using physicsbased description of the molecular processes accurately reproduced the first two congression phases. The results suggest that phase $\mathrm{i}$ corresponds to the time needed for MTs to find their partner SPB, and phase ii starts when Kar3 engages with MTs from the opposite SPB. Nuclear

Figure 7. Physics-based simulation of nuclear congression using Cytosim. (A) Cartoon of the three different models used to simulate nuclear congression. Green circles indicate the possible localizations of Kar3 in the different models. $(B)$ Snapshot of a simulation (model 1) $1 \mathrm{sec}$ after cell fusion. In the simulations, the MTs can grow (green arrowhead), undergo catastrophe, and shrink (red arrowhead). (C) Plots showing the distance between the two SPBs in mating cells from experiments and simulations. Thick solid lines show the median distances, and shaded areas indicate the upper and lower quartiles. The average of 100 simulations each is shown for each model. In model 1, Kar3 motors localize only at the SPB (Supplemental Movie S8). In model 2, the motors are present at the SPBs and on MT plus ends (green) (Supplemental Movie S9). For MT-localized motors, this model also contained, in addition to the SPB-bound motors, 2000 freely diffusing Kar3/Cik1 complexes with a binding activity to growing MT plus ends and the MT lattice via the nonmotor and motor domains. This resulted in $4.67 \pm 1.65$ motors per growing MT. Model 3 corresponds to model 2 without SPB-bound Kar3/ Cik1 complexes (red) (Supplemental Movie S10). For more information and parameters, see the Materials and Methods and Supplemental Table S1. Experimental data show wild-type (WT) SPB congression (black; from Fig. 2C). (D) Results for model 1 simulations containing different numbers of SPB-bound motors (140 [blue solid line] and 280 motors per SPB [blue dashed line]), and experimental data show CNM67-GFP-KAR3 kar34 SPBs congression (black; from Fig. 2F). 
congression then proceeds with a constant speed determined by the speed of Kar3. Our results show that MTs interacting with motors anchored at the opposite SPB are indeed a valid scenario for nuclear congression. We identified that plus end-localized Kar3/Cik1 can shorten the first search phase, but these interactions appear to be not sufficient to promote congression.

\section{Discussion}

The MT machinery is specifically regulated to perform nuclear congression. While it was well established that Kar3 together with Cik1 act on cytoplasmic MTs to drive nuclear migration, the limited resolution of light microscopy left several possibilities open for the underlying functional organization. The two pre-existing models (Rose 1996; Molk et al. 2006), based on the reported localization of Kar3 on MTs and/or at MT plus ends, involved direct MT-MT interactions. These models also satisfied the asymmetric mating defect of kar1 mutants caused by detachment of the MTs from one of the SPBs during mating (Rose and Fink 1987; Vallen et al. 1992; Pereira et al. 1999). Our initial observation showing that no clear MT-MT interactions could be detected was in apparent conflict with this kar1 phenotype, but this was resolved with the discovery that the Kar3/Cik1 localization to the SPB is dependent on an interaction with Spc72. Thus, Kar1 binding of Spc72 in mating cells recruits a functional bipartite MT-organizing module for MT nucleation and minus end binding via interaction of the $\mathrm{N}$-terminal domain with the $\gamma$-tubulin complex (Knop and Schiebel 1998) and via binding to Kar3/Cik1 (Fig. 3) to move on MTs from the opposite SPB. This suggests a simple model in which a mating-specific module of Kar1/Spc72/Kar3/Cik1 functionalizes the half bridge of the SPB to take a lead in congression. The nucleus, which is connected to the SPB via the nuclear membrane and the attached chromosomes, follows this movement up to the point at which two SPBs of the mating partner encounter each other and nuclear fusion is initiated.

Involved in multiple cellular functions at different developmental stages, Kar3 illustrates how the motor protein can be used differently by regulating the expression of associated proteins. In its different functions, Kar3 is associated with Cik1 or Vik1, thus forming distinct complexes (Manning et al. 1999). Transcriptional regulation of Cik1 into a cytoplasmic form upon stimulation of the mating process (Benanti et al. 2009) appears to be sufficient to adjust the localization and thus the functional activity of the motor. In mitotic spindles, Kar3 localizes on the nuclear side of the SPB (Zeng et al. 1999) in a Vik1dependent manner and in a Cik1-dependent manner on the plus ends of the nuclear interpolar MTs /Gardner et al. 2008). During mating, Vik1 is not expressed, and Kar3 interacts only with Cik1 (Manning et al. 1999). In shmooing cells, cytoplasmic Kar3/Cik1 has been proposed to maintain depolymerizing MT plus ends at the shmoo tip (Meluh and Rose 1990; Maddox et al. 2003). This is likely due to an interaction of Kar3 with the mating-specific $\mathrm{G} \alpha$ subunit of a trimeric $\mathrm{G}$ protein involved in pheromone signaling (Zaichick et al. 2009). Pheromone stimulation increases the expression of KAR3 and CIK1 via activation of the transcription factors Ste12 and Kar4 (Kurihara et al. 1996; Gammie et al. 1999; Lahav et al. 2007) and underlies the expression of the shorter NLS-less cytoplasmic Cik1 variant (Manning et al. 1999; Benanti et al. 2009). Thus, the cytoplasmic localization of Cik1 targets Kar3 to its cytoplasmic binding sites (i.e., the MT plus ends) (Sproul et al. 2005) and, as shown in this study, the cytoplasmic side of the SPB through a Cik1-codependent interaction of its $\mathrm{N}$-terminal domain with Spc72. Since Spc72 itself relocalizes to the half bridge during mating (Pereira et al. 1999), this leads to the reorganization of the entire cytoplasmic MT upon pheromone stimulation. At present, it is unclear how Spc72 binding to the SPB is regulated; one might speculate about a direct regulation of the Nud1/Spc72 and/or Kar1/Spc72 interactions by kinases involved in pheromone signaling. Altogether, this sets the stage to transport the SPB-and with it, the attached nucleus-along MTs from the opposite SPB. This seems to be sufficient to promote nuclear congression in an asymmetric manner (MTs anchored at one SPB, while the other SPB is sliding along these MTs) (Fig. 5). In wild-type cells, the process is symmetric, as both SPBs fulfill both roles simultaneously, increasing the overall robustness.

The realism of this model was proven, as nuclear congression occurred in silico with the very similar characteristics measured for the in vivo process. Nonetheless, functions that per se are not essential for congression may provide additional robustness to the process in vivo. First, the persistence of polarized actin cables could contribute to maintain the nucleus and the MTs orientated, such as the two SPBs remain facing each other until the MTs reach their opposite SPBs. Second, Kar3 is present also on the MT lattice, and its reported spindle function to regulate anti-parallel overlaps could promote such interactions also in karyogamy, as previously assumed (Rose 1996). Similarly, Kar3 present at the MT plus ends could still promote direct interactions between plus ends, as suggested by the "plus end interaction model" (Molk et al. 2006). The fact that we did not observe such MT-MT interactions in the limited numbers of tomograms that we generated does not mean that they never exist, but rather that they might be transient, occurring probably early in the process when these interactions may be promoting directional growth of MT toward opposite SPBs. The simulations indeed showed that direct Kar3mediated MT-MT interactions could shorten the initial search phase and make the migration more robust by helping MTs find the opposite SPB.

Altogether, our data argue for a system of striking elegance in which only a few molecules are involved, but they are arranged in what seems to be the best possible configuration considering the task at hand. Moreover, the manner in which they promote direct transport of nuclei along MTs is similar to nuclear migration in higher cells.

In contrast to yeast, the animal centrosome is not passively embedded in the nuclear envelope but exists as 
a dynamic organelle in the cytoplasm (Gönczy et al. 1999; Morris 2003). In this context, the movement of nuclei often relies on the attachment of the nucleus to a polarized MT array through MT motor proteins. This is the case in mammalian epithelial cells in which kinesin 1 at the nuclear surface moves nuclei toward plus ends of polarized MTs to the base of the cell (Bacallao et al. 1989; Roux et al. 2009). Similar mechanisms are involved in Xenopus female pronuclear movement, where the dyneincarrying female pronucleus tracks along MTs from the male-derived centrosome, thus pulling the two pronuclei together (Reinsch and Karsenti 1997). The yeast nuclear congression process described here is strikingly similar. The nucleus is transported via the minus end-directed kinesin-14 motor Kar3 along an array of MT nucleated by the SPB of the opposite nucleus, independently of its own SPB nucleation properties. Interestingly, in yeast, the motors are only localized at the SPB and not all over the surface of the nucleus. The reason for this is likely to be a functional one: While in animal cells, bringing the nuclei together in any orientation is probably sufficient, in yeast, the correct orientation of the nuclei such that the two SPBs are able to meet and fuse seems to be a prerequisite. The different functional requirements are again reflected by the different molecular players: the SPB components in yeast and SUN-KASH bridges in animal cells (Starr and Fridolfsson 2010).

\section{Materials and methods}

\section{Strains and yeast methods}

All yeast strains used in this study are derivatives of the S288c strains ESM356-1 (MATa) and ESM357-9 (MAT $\alpha$ ) (Knop and Schiebel 1998). The genotypes of the yeast strains used in this work are listed in Supplemental Table S2. The plasmids used in this study are listed in Supplemental Table S3. Basic yeast methods and growth medium are described elsewhere (Sherman 1991). Chromosomal manipulations of yeast strains (C-terminal tagging, gene deletions, and promoter substitutions) were performed using PCR-amplified cassettes as described previously (Janke et al. 2004). The N-terminal tagging of KAR3 was performed using seamless gene tagging by endonuclease-driven homologous recombination (Khmelinskii et al. 2011). Quantitative mating essays were preformed essentially as described (Pereira et al. 1999). Two-hybrid interactions were determined using plasmids and strains described earlier (Geissler et al. 1996; Schramm et al. 2000).

\section{Electron tomography}

Cells of both mating types were grown in log phase in YPD medium $\left(\sim 0.5 \times 10^{7}\right.$ cells per milliliter $)$ and mixed in equal proportions. Mixed cells were collected onto a nitrocellulose membrane $(0.45-\mu \mathrm{m}$ pore size; Millipore) using vacuum filtration and placed on a YPD agar plate for $2 \mathrm{~h}$ at $30^{\circ} \mathrm{C}$. Mating cells were cryoimmobilized by high-pressure freezing with a Leica EMPACT-2 (Leica Microsystems). Samples were then freezesubstituted and plastic-embedded as described previously (Höög and Antony 2007) using $0.2 \%$ uranyl acetate, $0.1 \%$ glutaraldehyde, and $1 \%$ water in anhydrous acetone as a freeze substitution solution. Serial thick sections (300-320 nm thick) were cut using a Reichert Ultracut-E microtome (Leica Microsystems) and collected on Formvar-coated palladium-copper slot grids. Post-staining was performed with $4 \%$ uranyl acetate in $70 \%$ methanol for $10 \mathrm{~min}$ and subsequently with lead citrate $(1.5 \%$ at $\mathrm{pH}$ 12) for $3 \mathrm{~min}$. Tilted images were acquired at $300 \mathrm{kV}$ from $-60^{\circ} \mathrm{C}$ to $+60^{\circ} \mathrm{C}$ with $1^{\circ} \mathrm{C}$ increments on a Tecnai $\mathrm{F} 30$ electron tomography microscope equipped with an Eagle 4K CCD camera (FEI: pixel size $1.499 \mathrm{~nm}$ at 15,500 magnification). Tomograms were then generated by R-weighted back projection, modeled, and analyzed using IMOD software as already described (Kremer et al. 1996).

\section{Live-cell fluorescent microscopy}

For imaging of mating yeast cells, cells of both mating types were grown in synthetic complete (SC)-complete medium to log phase $\left(\sim 0.5 \times 10^{7}\right.$ cells per milliliter $)$ and mixed together in equal proportions. Mixed cells were immobilized in glassbottomed well chambers (LabTek 155411; Nalce Nunc International). The chambers were pre-treated for at least $30 \mathrm{~min}$ with a 1:100 dilution of Bioconext in ethanol (UCT) followed by one ethanol and one water wash step, then incubated for $>30 \mathrm{~min}$ with 5\% Concanavalin A in water (C2010, Sigma) followed again by two washing steps with water. Following $1.5-2 \mathrm{~h}$ of incubation to allow mating to proceed, imaging was performed on a Deltavision RT wide-field microscope (Applied Precision) equipped with a uPlanApo $100 \times$ NA 1.35 oil immersion objective (Olympus), softWoRx software (Applied Precision), and a CoolSNAP HQ camera (Photometrics). For $\alpha$-factor treatment, $M A T a$ cells were grown in $\log$ phase in liquid SC medium and then immobilized in glass-bottomed well chambers, and $\alpha$-factor was added to a final concentration of $10 \mu \mathrm{g} / \mathrm{mL}$. Shmooing cells were imaged after $1.5-2 \mathrm{~h}$ of stimulation using the Deltavision RT microscope. Z-stacks (six planes at $0.5-0.6 \mu \mathrm{m}$ interval, $1 \times 1$ binning) for each channel (GFP and mCherry) and a single plane bright-field image were acquired every $10 \mathrm{sec}$ for 3-5 min. All movies show maximum projections of the Z-stacks at 10 frames per second (fps). For the purpose of visualization, images have been bleach-corrected by normalizing the intensities to the values at the beginning of the movie, and a Gaussian filter with a radius of 1 was applied.

\section{FRAP}

All imaging was performed at $30^{\circ} \mathrm{C}$ on a PerkinElmer Improvision Ultraview VoX spinning-disk confocal microscope equipped with a $100 \times 1.3$ NA Plan immersion oil objective. GFP was excited using the $488-\mathrm{nm}$ line of a $100-\mathrm{mW}$ argon laser at $10 \%$ laser power (Spectra Physics). A prebleach image was captured followed by bleaching of a circular area of $700 \mathrm{~nm}$ in diameter (four pulses of $1 \mathrm{msec}$ of the $488-\mathrm{nm}$ laser set to $60 \%$ intensity) followed by capturing of post-bleach images every $3 \mathrm{sec}$ for 100 $\mathrm{sec}$ (exposure ,150 msec; binning, 2). Six focal planes at $0.7-\mu \mathrm{m}$ distance were captured using a Hamamatsu C9100-50 camera. The centroid of the GFP-Kar3 spot has been tracked using the tracking algorithm from Sbalzarini and Koumoutsakos (2005). Fitting of a Gaussian to the fluorescent signal yielded a standard deviation $\sigma$ of $\sim 1.5$ pixels. The region of interest was taken in the $2 \sigma$ limit. We thus computed the intensity of an area of $6 \times 6$ pixels around the centroids of the bleached SPBs. This has been bleach-corrected using the total GFP-Kar3 fluorescence of other cells in the field of view as a reference.

\section{Protein localization at the SPB}

Cells were imaged on a Zeiss LSM 780 using a $63 \times 1.4$ NA PlanApo immersion oil objective at $30^{\circ} \mathrm{C}$. GFP and mCherry 
were excited simultaneously with a 488-nm argon laser and a 561-nm DPSS laser, respectively. The spectral array detector was used to image the green and red channels simultaneously. We acquired 26 planes with $0.2-\mu \mathrm{m}$ distance. The position of the proteins at the SPB was determined by finding the peak of a Gaussian fitted to the fluorescence signal in the plane with maximal intensity. The distal position of each nucleus with respect to Spc42-mCherry served as a reference coordinate for the nucleus.

\section{Quantification of GFP-Kar3 at the SPB and measurement of nuclear congression kinetics}

Images were acquired using a wide-field Olympus IX81 with a $100 \times 1.4$ NA PlanApo oil objective and a Hamamatsu OrcaER camera (binning of 2; pixel size, $0.13 \mu \mathrm{m}$ ). For the quantification of the GFP signal of GFP-Kar3, MATa- and MAT $\alpha$ GFP-KAR3expressing cells were mixed and allowed to mate as described before. After 1.5 h, MATa NUF2-GFP PIL1-mCherry-expressing cells were added to the glass-bottomed well chambers and allowed to settle for $10 \mathrm{~min}$. The Pill-mCherry signal was used to identify these cells. Per field of view, 31 planes at a $0.2-\mu \mathrm{m}$ distance (200 msec exposure for GFP, $100 \mathrm{msec}$ for mCherry) were acquired. Quantification was done as described by Joglekar et al. (2006). The number of GFP molecules present in the GFPKar3 spot was calculated by comparison with the Nuf2-GFP spot in metaphase cells, which contains on average 385.6 molecules of GFP (Lawrimore et al. 2011).

To determine the SPBs' nuclear congression dynamics, cells of both mating types expressing SPC42-GFP (or CNM67-GFPKAR3) and mCherry-TUB1 were used. To determine the time point of cell fusion, one mating partner additionally expressed soluble cytoplasmic TagBFP from the GPD promoter. Congression speed was determined from the slope of a line fitted to five consecutive data points during congression. Images, five planes with a spacing of $0.5 \mu \mathrm{m}$, were taken every $20 \mathrm{sec}$. Tracking of the position of the SPBs was conducted as described above.

\section{First principles modeling of MT and nuclear dynamics}

In silico nuclear congression simulations were carried out using Cytosim, a program developed for stochastic simulations of cytoskeletal fibers, diffusible activities, and flexible objects in a confined environment. The simulation is based on Brownian dynamics, and the system properties emerge from real amounts of constituents following a stochastic description of the most relevant physical processes (Nedelec and Foethke 2007). The nucleus is spherical, but the SPB is another smaller sphere attached on its side with three Hookean springs. This setup represents the nuclear protrusions observed in the tomograms. The MTs are anchored at the SPB and have a restricted rotational freedom at their SPB attachment point so that, on average, they grow away from the nucleus from which they were nucleated. MTs are modeled as dynamic growing fibers that stochastically switch to shrinkage. Below a minimal length, MTs are rescued and switch back to growth. In the model, we restrict ourselves to the situation just after cell fusion and assume that nuclei and MTs are properly aligned toward the shmoo as observed (Maddox et al. 2003). The initial distance of SPB to neck was $3.08 \pm 0.61$ $\mu \mathrm{m}$ for wild type and $2.4 \pm 0.54 \mu \mathrm{m}$ for CNM67-GFP-KAR3 kar3s to match the measured distances at cell fusion. MTs were straight in the growing or shrinking states with equal probability, and their lengths were randomly distributed with the constraint that they should fit in the cell before fusion. The model parameters for the dynamics of MTs, the Kar3 minus end-directed motors, and the geometry of the mating cells have been obtained from the literature, the tomograms presented here, and the dynamics of GFP-Kar3 during congression (Supplemental Table S1). To simulate the MT signal in light microscopy, we approximated the point spread function (PSF) of the microscope with a Gaussian of width

$$
\sigma_{x y}=\sqrt{(0.25 \lambda / N A)^{2}+1 / 12 a^{2}}
$$

where $N A=1.4$ is the numerical aperture, $a=0.13 \mu \mathrm{m}$ is the pixel size, and $\lambda$ is the emission wavelength (Mortensen et al. 2010; Stallinga and Rieger 2010). Model fluorophores, uniformly distributed on MTs, are convolved with this function.

\section{Acknowledgments}

We thank the members of the Antony, Knop, and Nédélec groups and the EMBL Electron Microscopy Core Facility for helpful discussions and technical support. We acknowledge the people in the Nédélec group involved in the development of Cytosim. Kornelius Schweinfurth is acknowledged for help in tomography modeling. We thank Susanne Trautmann, Celine Maeder, and Matthias Meurer for plasmids. We acknowledge Tomo Tanaka for discussions. We are grateful to Elmar Schiebel for plasmids, discussion, and comments on the manuscript. We also thank Charlotta Funaya for critical reading. A.Z.P. is supported by a post-doctoral grant from BIOMS (Center for Modelling and Simulation in the Biosciences in Heidelberg). C.A., M.K. and F.N. designed the project. R.G. and A.Z.P. together did the light microscopy experiments and image analysis. R.G. performed yeast genetic experiments and electron microscopy, and A.Z.P. performed the first principles modeling. M.K. and R.G., helped by A.Z.P., wrote the manuscript with input from C.A. and F.N. All authors discussed the results and commented on the manuscript.

\section{References}

Allingham JS, Sproul LR, Rayment I, Gilbert SP. 2007. Vik1 modulates microtubule-Kar3 interactions through a motor domain that lacks an active site. Cell 128: 1161-1172.

Bacallao R, Antony C, Dotti C, Karsenti E, Stelzer EH, Simons K. 1989. The subcellular organization of Madin-Darby canine kidney cells during the formation of a polarized epithelium. J Cell Biol 109: 2817-2832.

Barrett JG, Manning BD, Snyder M. 2000. The Kar3p kinesinrelated protein forms a novel heterodimeric structure with its associated protein Cik1p. Mol Biol Cell 11: 2373-2385.

Benanti JA, Matyskiela ME, Morgan DO, Toczyski DP. 2009. Functionally distinct isoforms of Cik1 are differentially regulated by APC/C-mediated proteolysis. Mol Cell 33: 581-590.

Byers B, Goetsch L. 1975. Behavior of spindles and spindle plaques in the cell cycle and conjugation of Saccharomyces cerevisiae. I Bacteriol 124: 511-523.

Chen CJ, Rayment I, Gilbert SP. 2011. Kinesin Kar3Cik1 ATPase pathway for microtubule cross-linking. I Biol Chem 286: 29261-29272.

Chu HM, Yun M, Anderson DE, Sage H, Park HW, Endow SA. 2005. Kar3 interaction with Cik1 alters motor structure and function. $E M B O$ I 24: 3214-3223.

Conde J, Fink GR. 1976. A mutant of Saccharomyces cerevisiae defective for nuclear fusion. Proc Natl Acad Sci 73: 36513655.

Elliott S, Knop M, Schlenstedt G, Schiebel E. 1999. Spc29p is a component of the Spc110p subcomplex and is essential for 
spindle pole body duplication. Proc Natl Acad Sci 96: 62056210.

Endow SA, Kang SJ, Satterwhite LL, Rose MD, Skeen VP, Salmon ED. 1994. Yeast Kar3 is a minus-end microtubule motor protein that destabilizes microtubules preferentially at the minus ends. $E M B O J$ 13: 2708-2713.

Gammie AE, Stewart BG, Scott CF, Rose MD. 1999. The two forms of karyogamy transcription factor Kar4p are regulated by differential initiation of transcription, translation, and protein turnover. Mol Cell Biol 19: 817-825.

Gardner MK, Haase J, Mythreye K, Molk JN, Anderson M, Joglekar AP, O'Toole ET, Winey M, Salmon ED, Odde DI, et al. 2008. The microtubule-based motor Kar3 and plus endbinding protein Bim1 provide structural support for the anaphase spindle. J Cell Biol 180: 91-100.

Geissler S, Pereira G, Spang A, Knop M, Souès S, Kilmartin J, Schiebel E. 1996. The spindle pole body component Spc98p interacts with the $\gamma$-tubulin-like Tub4p of Saccharomyces cerevisiae at the sites of microtubule attachment. EMBO $J$ 15: 3899-3911.

Gönczy P, Pichler S, Kirkham M, Hyman A. 1999. Cytoplasmic dynein is required for distinct aspects of MTOC positioning, including centrosome separation, in the one cell stage Caenorhabditis elegans embryo. J Cell Biol 147: 135-150.

Grueneberg U, Campbell K, Simpson C, Grindlay J, Schiebel E. 2000. Nudlp links astral microtubule organization and the control of exit from mitosis. EMBO J 19: 6475-6488.

Höög JL, Antony C. 2007. Whole-cell investigation of microtubule cytoskeleton architecture by electron tomography. Methods Cell Biol 79: 145-167.

Howard J. 1996. The movement of kinesin along microtubules. Annu Rev Physiol 58: 703-729.

Janke C, Magiera MM, Rathfelder N, Taxis C, Reber S, Maekawa H, Moreno-Borchart A, Doenges G, Schwob E, Schiebel E, et al. 2004. A versatile toolbox for PCR-based tagging of yeast genes: New fluorescent proteins, more markers and promoter substitution cassettes. Yeast 21: 947-962.

Jin F, Liu H, Li P, Yu H-G, Wang Y. 2012. Loss of function of the Cik1/Kar3 motor complex results in chromosomes with syntelic attachment that are sensed by the tension checkpoint. PLoS Genet 8: e1002492.

Joglekar AP, Bouck DC, Molk JN, Bloom KS, Salmon ED. 2006. Molecular architecture of a kinetochore-microtubule attachment site. Nat Cell Biol 8: 581-585.

Khmelinskii A, Meurer M, Duishoev N, Delhomme N, Knop M. 2011. Seamless gene tagging by endonuclease-driven homologous recombination. PLOS ONE 6: e23794.

Knop M, Schiebel E. 1997. Spc98p and Spc97p of the yeast $\gamma$-tubulin complex mediate binding to the spindle pole body via their interaction with Spc110p. EMBO J 16: 6985-6995.

Knop M, Schiebel E. 1998. Receptors determine the cellular localization of a $\gamma$-tubulin complex and thereby the site of microtubule formation. EMBO J 17: 3952-3967.

Kremer JR, Mastronarde DN, McIntosh JR. 1996. Computer visualization of three-dimensional image data using IMOD. I Struct Biol 116: 71-76.

Kurihara LJ, Stewart BG, Gammie AE, Rose MD. 1996. Kar4p, a karyogamy-specific component of the yeast pheromone response pathway. Mol Cell Biol 16: 3990-4002.

Lahav R, Gammie AE, Tavazoie S, Rose MD. 2007. Role of transcription factor Kar4 in regulating downstream events in the Saccharomyces cerevisiae pheromone response pathway. Mol Cell Biol 27: 818-829.

Lawrimore J, Bloom KS, Salmon ED. 2011. Point centromeres contain more than a single centromere-specific Cse4 (CENP-A) nucleosome. J Cell Biol 195: 573-582.
Maddox PS, Stemple JK, Satterwhite LL, Salmon ED, Bloom KS. 2003. The minus end-directed motor Kar3 is required for coupling dynamic microtubule plus ends to the cortical shmoo tip in budding yeast. Curr Biol 13: 1423-1428.

Manning BD, Barrett JG, Wallace JA, Granok H, Snyder M. 1999. Differential regulation of the Kar3p kinesin-related protein by two associated proteins, Cik1p and Vik1p. J Cell Biol 144: 1219-1233.

Melloy P, Shen S, White E, McIntosh JR, Rose MD. 2007. Nuclear fusion during yeast mating occurs by a three-step pathway. J Cell Biol 179: 659-670.

Melloy P, Shen S, White E, Rose MD. 2009. Distinct roles for key karyogamy proteins during yeast nuclear fusion. Mol Biol Cell 20: 3773-3782.

Meluh PB, Rose MD. 1990. KAR3, a kinesin-related gene required for yeast nuclear fusion. Cell 60: 1029-1041.

Mogilner A, Craig E. 2010. Towards a quantitative understanding of mitotic spindle assembly and mechanics. I Cell Sci 123: 3435-3445.

Molk JN, Bloom KS. 2006. Microtubule dynamics in the budding yeast mating pathway. J Cell Sci 119: 3485-3490.

Molk JN, Salmon ED, Bloom KS. 2006. Nuclear congression is driven by cytoplasmic microtubule plus end interactions in S. cerevisiae. J Cell Biol 172: 27-39.

Morris NR. 2003. Nuclear positioning: The means is at the ends. Curr Opin Cell Biol 15: 54-59.

Mortensen KI, Churchman LS, Spudich JA, Flyvbjerg H. 2010. Optimized localization analysis for single-molecule tracking and super-resolution microscopy. Nat Methods 7: 377-381.

Muller EGD, Snydsman BE, Novik I, Hailey DW, Gestaut DR, Niemann CA, O'Toole ET, Giddings TH, Sundin BA, Davis TN. 2005. The organization of the core proteins of the yeast spindle pole body. Mol Biol Cell 16: 3341-3352.

Nedelec F, Foethke D. 2007. Collective Langevin dynamics of flexible cytoskeletal fibers. New J Phys 9: 427.

Page BD, Snyder M. 1992. CIK1: A developmentally regulated spindle pole body-associated protein important for microtubule functions in Saccharomyces cerevisiae. Genes Dev 6: 1414-1429.

Page BD, Satterwhite LL, Rose MD, Snyder M. 1994. Localization of the Kar3 kinesin heavy chain-related protein requires the Cik1 interacting protein. J Cell Biol 124: 507-519.

Pereira G, Grueneberg U, Knop M, Schiebel E. 1999. Interaction of the yeast $\gamma$-tubulin complex-binding protein Spc72p with Karlp is essential for microtubule function during karyogamy. EMBO J 18: 4180-4195.

Polaina J, Conde J. 1982. Genes involved in the control of nuclear fusion during the sexual cycle of Saccharomyces cerevisiae. Mol Gen Genet 186: 253-258.

Reinsch S, Karsenti E. 1997. Movement of nuclei along microtubules in Xenopus egg extracts. Curr Biol 7: 211-214.

Rose MD. 1996. Nuclear fusion in the yeast Saccharomyces cerevisiae. Annu Rev Cell Dev Biol 12: 663-695.

Rose MD, Fink GR. 1987. KAR1, a gene required for function of both intranuclear and extranuclear microtubules in yeast. Cell 48: 1047-1060.

Roux KJ, Crisp ML, Liu Q, Kim D, Kozlov S, Stewart CL, Burke B. 2009. Nesprin 4 is an outer nuclear membrane protein that can induce kinesin-mediated cell polarization. Proc Natl Acad Sci 106: 2194-2199.

Sbalzarini IF, Koumoutsakos P. 2005. Feature point tracking and trajectory analysis for video imaging in cell biology. I Struct Biol 151: 182-195.

Schramm C, Elliott S, Shevchenko A, Schiebel E. 2000. The Bbplp-Mps2p complex connects the SPB to the nuclear 
envelope and is essential for SPB duplication. $E M B O J \mathbf{1 9}$ 421-433.

Shanks RMQ, Kamieniecki RJ, Dawson DS. 2001. The Kar3interacting protein Ciklp plays a critical role in passage through meiosis I in Saccharomyces cerevisiae. Genetics 159: 939-951.

Shanks RMQ, Bascom-Slack C, Dawson DS. 2004. Analysis of the kar3 meiotic arrest in Saccharomyces cerevisiae. Cell Cycle 3: 363-371.

Sherman F. 1991. Getting started with yeast. Methods Enzymol 194: $3-21$.

Souès S, Adams IR. 1998. SPC72: A spindle pole component required for spindle orientation in the yeast Saccharomyces cerevisiae. J Cell Sci 111: 2809-2818.

Spang A, Courtney I, Grein K, Matzner M, Schiebel E. 1995. The Cdc31p-binding protein Karlp is a component of the half bridge of the yeast spindle pole body. I Cell Biol 128: 863877.

Sproul LR, Anderson DJ, Mackey AT, Saunders WS, Gilbert SP. 2005. Cik1 targets the minus-end kinesin depolymerase kar3 to microtubule plus ends. Curr Biol 15: 1420-1427.

Stallinga S, Rieger B. 2010. Accuracy of the Gaussian point spread function model in 2D localization microscopy. Opt Express 18: 24461-24476.

Starr DA, Fridolfsson HN. 2010. Interactions between nuclei and the cytoskeleton are mediated by SUN-KASH nuclearenvelope bridges. Annu Rev Cell Dev Biol 26: 421-444.

Vallen EA, Hiller MA, Scherson TY, Rose MD. 1992. Separate domains of KAR1 mediate distinct functions in mitosis and nuclear fusion. J Cell Biol 117: 1277-1287.

Verhey KJ, Kaul N, Soppina V. 2011. Kinesin assembly and movement in cells. Annu Rev Biophys 40: 267-288.

Zaichick SV, Metodiev MV, Nelson SA, Durbrovskyi O, Draper E, Cooper JA, Stone DE. 2009. The mating-specific G $\alpha$ interacts with a kinesin-14 and regulates pheromoneinduced nuclear migration in budding yeast. Mol Biol Cell 20: $2820-2830$.

Zeng X, Kahana JA, Silver PA, Morphew MK, McIntosh JR, Fitch IT, Carbon J, Saunders WS. 1999. Slk19p is a centromere protein that functions to stabilize mitotic spindles. I Cell Biol 146: 415-425. 


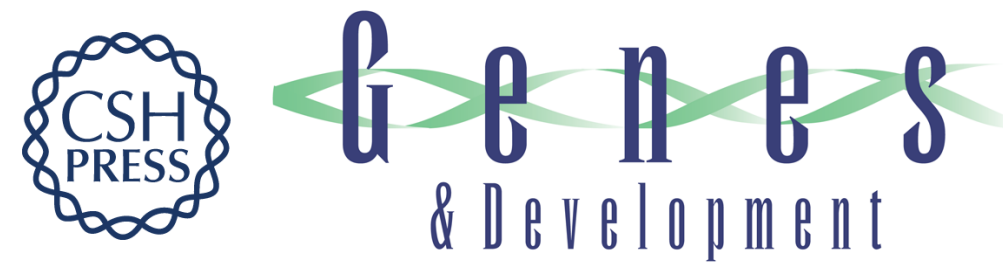

\section{Spindle pole body-anchored Kar3 drives the nucleus along microtubules from another nucleus in preparation for nuclear fusion during yeast karyogamy}

Romain Gibeaux, Antonio Z. Politi, François Nédélec, et al.

Genes Dev. 2013, 27:

Access the most recent version at doi:10.1101/gad.206318.112

Supplemental http://genesdev.cshlp.org/content/suppl/2013/02/06/27.3.335.DC1

Material

References This article cites 61 articles, 30 of which can be accessed free at: http://genesdev.cshlp.org/content/27/3/335.full.html\#ref-list-1

License

Email Alerting Receive free email alerts when new articles cite this article - sign up in the box at the top Service right corner of the article or click here.

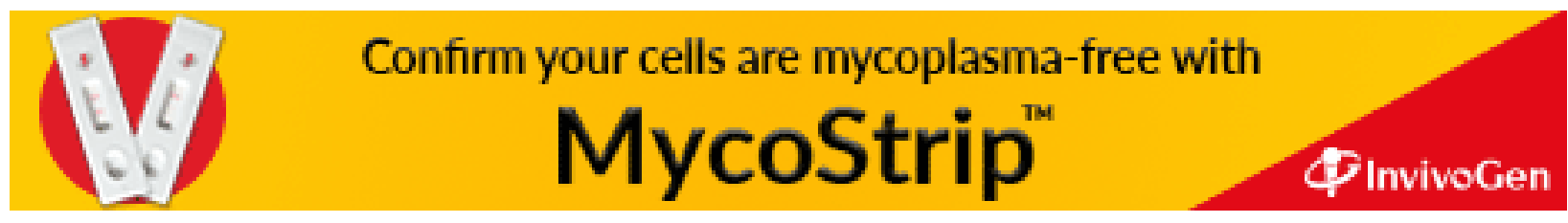

